\title{
EXIT Charts for System Design and Analysis
}

\author{
Mohammed El-Hajjar and Lajos Hanzo
}

\begin{abstract}
Near-capacity performance may be achieved with the aid of iterative decoding, where extrinsic soft information is exchanged between the constituent decoders in order to improve the attainable system performance. Extrinsic Information Transfer (EXIT) charts constitute a powerful semi-analytical tool used for analysing and designing iteratively decoded systems. In this tutorial, we commence by providing a rudimentary overview of the iterative decoding principle and the concept of soft information exchange. We then elaborate on the concept of EXIT charts using three iteratively decoded prototype systems as design examples. We conclude by illustrating further applications of EXIT charts, including near-capacity designs, the concept of irregular codes and the design of modulation schemes.
\end{abstract}

Index Terms-EXIT charts, convolutional codes, turbo codes, concatenated codes, mutual information.

\section{INTRODUCTION}

$\mathbf{I}$ $\mathrm{N}$ THE ERA of smart phones and tablet PCs, there is a demand for flawless high-rate wireless multimedia services. On the other hand, bandwidth is a valuable commodity and therefore the information has to be transmitted over the communication channel as efficiently as possible due to the bandlimited nature of communication networks $[1,2]$. Furthermore, the capacity of wireless systems is interference limited, hence cannot be readily increased by simply increasing the transmitted power [2]. Therefore, flexible and bandwidth-efficient transceivers have to be designed for supporting wireless multimedia services.

The history of channel coding dates back to Shannon's pioneering work in $1948[3,4]$, where it was predicted that reliable communication can be achieved with the aid of channel coding by incorporating redundancy into the transmitted message. Ever since Shannon quantified the capacity of a communications system [3], researchers have endeavoured to devise high-speed, high-quality wireless communication systems exhibiting both a high bit rate and a low Bit Error Rate (BER). Advances in coding made it feasible to approach Shannon's capacity limit $[4,5]$. Channel coding dates back to the 1950s, when the first Hamming codes were invented [6]. Convolutional codes were introduced in [7] and then several algorithms were proposed for their decoding in [8-11]. Then Viterbi invented the Maximum Likelihood Sequence Estimation (MLSE) algorithm in 1967 [12,13], which was a major breakthrough for convolutional codes. In 1974, the Maximum A Posteriori (MAP) algorithm was proposed for attaining the minimum BER for the convolutional codes [14].

Manuscript received July 2, 2012; revised January 8, 2013 and March 15, 2013. The financial support of the RC-UK under the auspices of the IndiaUK Advanced Technology Centre (IU-ATC) and the EU's Concerto project is gratefully acknowledged.

The authors are with the School of Electronics and Computer Science, University of Southampton, SO17 1BJ, UK (e-mail: $\{$ meh,lh $\} @$ ecs.soton.ac.uk).

Digital Object Identifier 10.1109/SURV.2013.050813.00137
However, the MAP algorithm has not been widely employed until the invention of turbo codes, because its complexity was substantially higher than that of the Viterbi algorithm, whilst its performance was only marginally better.

The concept of concatenated codes was proposed in [15]. However, it was not until the turbo codes were proposed in [16, 17] that the concept of iterative decoding at low complexity became a reality with the employment of simple constituent codes. Turbo codes use parallel concatenation of two Recursive Systematic Convolutional (RSC) codes accommodating an interleaver between the two constituent encoders $[16,17]$. The concept of turbo codes was extended to multiple parallel concatenated codes in [18]. Then, the turbo principle was extended to both serially concatenated block and convolutional codes in [19]. In [20-22] the concept and design rules of BitInterleaved Coded Modulation (BICM) and BICM relying on Iterative Detection (BICM-ID) were presented.

Substantial efforts have been invested in optimising the performance of concatenated codes in order to improve the slopes of their BER curves and to attain a near-capacity performance. Recently, significant efforts have also been dedicated to studying the convergence behaviour of iterative decoding. Semi-analytical tools devised for analysing the convergence behaviour of iteratively decoded systems were proposed in [23-26, 28, 31,33-36,41-43, 45,46]. The Mutual Information (MI) between the data bits at the transmitter and the soft values at the receiver were used for describing the exchange of extrinsic information between the decoders in [47]. The exchange of extrinsic information between the constituent decoders may be visualised by EXtrinsic Information Transfer (EXIT) charts [23-25,46,48]. EXIT charts characterise the flow of information between the constituent decoders of a concatenated structure. In [36] the computation of EXIT charts was simplified for the scenario when the Probability Density Function (PDF) of the transmitted message is symmetric. An insightful tutorial introduction to EXIT charts was provided by Hagenauer in [49]. Additionally, several algorithms predicting the decoding convergence of iterative decoding schemes were compared in [35].

The concept of EXIT charts was further developed for analysing three-stage concatenated systems by Ten Brink in [26], Tüchler in [36] and Brannstrom in [42]. In [41,42] an algorithm was proposed for finding the optimal decoder activation order, namely for determining the order of softinformation exchange among the three components. Additionally, a beneficial technique was suggested in $[41,42]$ for projecting three-dimensional EXIT functions onto a single two-dimensional EXIT chart. Furthermore, it was proposed in $[37,38]$ to create systems exhibiting beneficial decoding convergence after a fixed number of iterations, which resulted 
TABLE I

MAJOR CONTRIBUTIONS ON STUDYING THE CONVERGENCE OF ITERATIVE DECODING.

\begin{tabular}{|c|c|c|}
\hline Year & Author(s) & Contribution \\
\hline$\overline{1999}$ & Peleg et al. [23] & $\begin{array}{l}\text { investigated the convergence behaviour of inner rate-1 codes based on a combination of Signal to Noise } \\
\text { Ratio (SNR) measures and mutual information. }\end{array}$ \\
\hline 2000 & ten Brink [24-26] & $\begin{array}{l}\text { proposed the employment of the EXIT charts for characterising the convergence behaviour of concatenated } \\
\text { decoders. }\end{array}$ \\
\hline 2001 & Richardson et al. [28] & $\begin{array}{l}\text { the density evolution algorithm was employed for the sake of constructing LDPC codes capable of } \\
\text { operating at low } E_{b} / N_{0} \text { values. }\end{array}$ \\
\hline 2001 & Chung et al. [29] & $\begin{array}{l}\text { the density evolution algorithm was also employed for the sake of designing LDPC codes operating } \\
\text { within } 0.0045 \mathrm{~dB} \text { of the Shannon limit. }\end{array}$ \\
\hline 2001 & ten Brink [30] & the EXIT chart analysis was extended to three-stage parallel concatenated systems. \\
\hline 2001 & El Gamal and Hammons [31] & SNR based measures were used for studying the convergence of iterative decoding. \\
\hline 2001 & Scanavino et al. [32] & $\begin{array}{l}\text { investigated the convergence properties of iterative decoders working at both bit and symbol level, where } \\
\text { useful performance improvements were observed, when employing symbol-based iterative decoding. }\end{array}$ \\
\hline 2001 & Grant [33] & $\begin{array}{l}\text { EXIT charts were extended to the non-binary (index-based) case, where a histogram-based approximation } \\
\text { of the extrinsic information was used in order to compute the mutual information. }\end{array}$ \\
\hline 2002 & Tüchler and Hagenauer [34] & $\begin{array}{l}\text { the computation of EXIT charts was further simplified to a time average, when PDFs of the communicated } \\
\text { information at the input and output of the constituent decoders are both symmetric and consistent. }\end{array}$ \\
\hline 2002 & Tüchler et al. [35] & several algorithms predicting the decoding convergence of iterative decoding schemes were compared. \\
\hline 2002 & Tüchler [36] & the EXIT chart analysis was extended to three-stage serially concatenated systems. \\
\hline 2003 & Tüchler $[37,38]$ & $\begin{array}{l}\text { a design procedure was proposed for creating systems exhibiting beneficial decoding convergence after } \\
\text { a fixed number of iterations. }\end{array}$ \\
\hline 2004 & Tüchler and Hagenauer $[34,38]$ & $\begin{array}{l}\text { Irregular Convolutional Codes (IrCC) were proposed for the sake of appropriately shaping the EXIT } \\
\text { curves by minimising the area within the EXIT-tunnel. }\end{array}$ \\
\hline 2004 & Lee et. al. $[39,40]$ & The EXIT band chart tool was proposed for analysing systems having short interleaver length. \\
\hline 2003-2005 & Brännström et al. $[41,42]$ & $\begin{array}{l}\text { the EXIT chart analysis of multiple concatenated codes was considered, where an algorithm was proposed } \\
\text { for finding the optimal decoder activation order. Additionally, a technique was also given for combining } \\
\text { and projecting a series of three-dimensional EXIT functions onto a single two-dimensional EXIT chart. }\end{array}$ \\
\hline 2006 & Kliewer et al. [43] & $\begin{array}{l}\text { an efficient and low-complexity method of computing non-binary EXIT charts from index-based a } \\
\text { posteriori probabilities was proposed, which may considered a generalisation of the approach presented } \\
\text { in [44]. }\end{array}$ \\
\hline
\end{tabular}

in a complexity reduction. The design procedure is based on the observation that EXIT chart predictions are usually accurately satisfied by the BER curves for the first few iterations, regardless of the depth of the interleaver employed.

The design of Irregular Convolutional Codes (IrCC) was proposed by Tüchler and Hagenauer in $[34,38]$ for the sake of accurately matching the shape of the EXIT curves of the decoder components by minimising the area within the open EXIT tunnel. On the other hand, Lee et. al. [39, 40] proposed the principle of EXIT-band charts as a tool for analysing systems having short interleaver length. The major contributions on studying the convergence of iterative decoding are summarised in Table I.

In this treatise we aim for providing a comprehensive tutorial of the semi-analytical EXIT chart tool used for designing and analysing iteratively decoded systems. The rest of the paper is organised as follows. In Section II, we present a brief overview of the iterative decoding principle followed by a tutorial insights into the employment of EXIT charts in Section III. Our discourse essentially relies on design examples. We then present further applications of EXIT charts in Sections IV and V. Finally, we conclude in Section VII.

\section{OVERVIEW OF CONCATENATED CODES AND THE TURBO PRINCIPLE}

The concatenation of low-complexity channel codes constitutes a convenient technique of constructing powerful codes capable of approaching the capacity, while having a moderate decoding complexity. Serially concatenated codes were proposed in 1966 in [15], while the discovery of parallel concate-
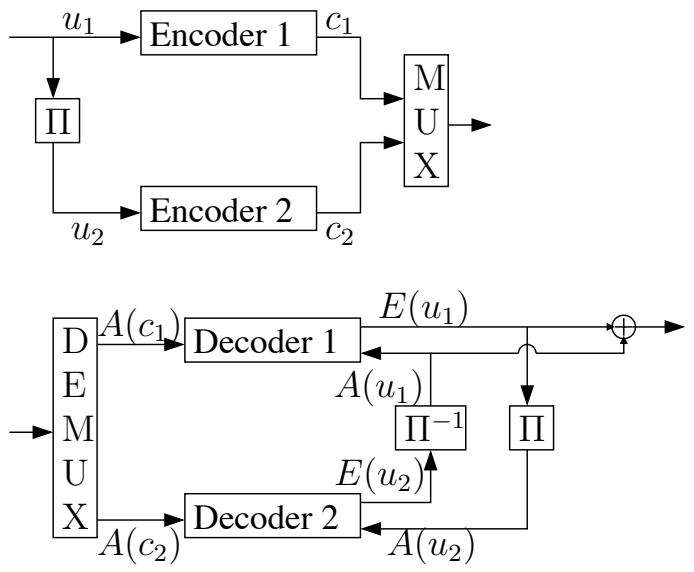

Fig. 1. Encoder and decoder structure of a parallel concatenated code [54].

nated codes [16] improved the achievable system performance by separating the constituent codes using interleavers for the sake of exchanging sufficient decorrelated extrinsic information between the constituent decoders in order to improve the system's BER [50]. Iterative decoding and the turbo principle [51] can be employed for both parallel concatenated codes [16-18] and serially concatenated codes $[19,52,53]$. In this section we provide a rudimentary overview of the different concatenated code schemes, including both parallel and serial concatenated structures.

The block diagram of parallel concatenated codes is shown in Fig. 1. As shown in Fig. 1 the input bit stream $\mathbf{u}_{\mathbf{1}}$ is encoded in Encoder 1 and an interleaved version of the input bit stream 

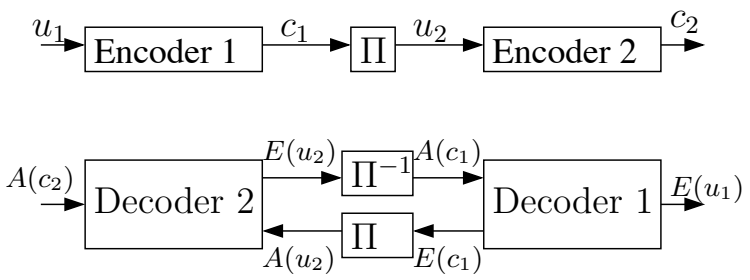

Fig. 2. Encoder and decoder structure of a two-stage serial concatenated code [54].

$\mathbf{u}_{\mathbf{2}}$ is encoded by Encoder 2. The role of this interleaver is that of ensuring that the parity-related redundancy appropriately inserted into the original information bit-stream is spread across a long sequence. The rationale of this is that this way each information bit is protected by parity bits, which are less likely to be affected by the same channel effects, as the information bits they are protecting. The output of the two encoders is then multiplexed and transmitted, as shown in Fig. 1.

At the receiver side, the two decoders, namely Decoder 1 and Decoder 2 of Fig. 1, exchange soft information in the form of Logarithmic-Likelihood Ratio (LLR), where the notations $A($.$) and E($.$) used in Fig. 1$ refer to the Extrinsic and $a$ priori LLRs of the bits, respectively. First, the received data is demultiplexed, as shown in Fig. 1 and then the LLRs representing the encoded bit streams $\mathbf{c}_{1}$ and $\mathbf{c}_{2}$ are passed to their corresponding decoders. Decoder 1 generates the extrinsic LLRs $E\left(\mathbf{u}_{\mathbf{1}}\right)$, as shown in Fig. 1, which are then interleaved to form the a priori LLRs $A\left(\mathbf{u}_{\mathbf{2}}\right)$ for Decoder 2 . To elaborate a little further, the bit providing a priori information from Decoder 2 would have travelled through the channel with a high time-displacement and hence is likely to be affected by statistically independent propagation phenomena. This results in time diversity.

Decoder 2 uses the a priori LLRs $A\left(\mathbf{c}_{\mathbf{2}}\right)$ and $A\left(\mathbf{u}_{\mathbf{2}}\right)$ in order to generate improved extrinsic LLRs $E\left(\mathbf{u}_{\mathbf{2}}\right)$, which are then de-interleaved and passed to Decoder 1 as a priori LLRs, as shown in Fig. 1. In other words, the interleaver/deinterleaver pair simply order the soft information in the correct order. Decoder 1 then uses the a priori LLRs for getting an improved extrinsic LLRs $E\left(\mathbf{u}_{\mathbf{1}}\right)$, which are then passed to Decoder 2 for further iterations.

Fig. 1 shows the block diagram of a non-systematic parallel concatenated code. It is possible to design a systematic parallel concatenated code, where in addition to encoding the input bit stream in encoders 1 and 2, the input bit stream is simply passed to the output. In the decoder of the systematic parallel concatenated codes, the extrinsic information is exchanged between the two constituent decoders, while also benefiting from the a priori LLRs provided for the systematic bits [54].

Fig. 1 shows a parallel concatenated code using two constituent codes. It is possible to use more than two constituent codes leading to multiple-stage turbo codes $[4,18,55]$. Additionally, the constituent codes used in turbo codes may be from any arbitrary channel code family, although Convolutional Codes (CC) are used most frequently.

On the other hand, iterative decoding may be applied to serially concatenated systems as shown in Fig. 2 and Fig. 3 for two-stage and three-stage concatenated systems, respectively. Fig. 2 shows the encoder and decoder block diagrams of a two-stage serially concatenated system consisting of an outer encoder, Encoder 1 of Fig. 2, followed by an interleaver connected to an inner encoder, Encoder 2 of Fig. 2. Again, the interleaver is used for introducing time-diversity by scrambling the bit sequence and hence providing independent a priori information, which is capable of reducing the effects of deep fades on the output of the outer decoder. As shown in Fig. 2, the input bit stream $\mathbf{u}_{1}$ is first encoded by Encoder 1 to generate the bit sequence $\mathbf{c}_{\mathbf{1}}$, which is then interleaved by interleaver $\Pi$ of Fig. 2. The interleaved bit stream $\mathbf{u}_{2}$ is then encoded by Encoder 2 to produce the bit stream $\mathbf{c}_{2}$. In the decoder of the two-stage serially concatenated codes, the $a$ priori LLRs $A\left(\mathbf{c}_{\mathbf{2}}\right)$ of bits $\mathbf{c}_{\mathbf{2}}$ are passed to Decoder 2, which outputs its extrinsic LLRs $E\left(\mathbf{u}_{2}\right)$. The output of Decoder 2 is then de-interleaved and passed to Decoder 1 as a priori LLRs, as shown in Fig. 2. Similarly to parallel concatenated codes, the role of the interleaver/de-interleaver pair is to ensure the correct ordering/alignment of the a priori information.

Decoder 1 then decodes the input soft information and output its extrinsic LLRs $E\left(\mathbf{c}_{\mathbf{1}}\right)$, which are then interleaved and passed back to Decoder 2 as a priori LLRs. Decoder 2 then uses its a priori LLRs input in order to provide improved extrinsic LLRs, which are passed to Decoder 1 and are then used for further iterations, as shown in Fig. 2. After the last iteration, Decoder 1 outputs the extrinsic LLRs for its data input stream $E\left(\mathbf{u}_{\mathbf{1}}\right)$, which can be passed to a hard decision decoder. In the receiver of the two-stage system shown in Fig. 2 iterative decoding is implemented by exchanging soft information between the two constituent decoders $[1,4,52,54]$. Over the years, the concept of iterative decoding of serially concatenated schemes was extended to turbo equalisation [4,56], coded modulation [21,57], multiuser detection [58] and to joint channel-estimation and datadetection plus synchronisation as well as joint source-channel decoding $[1,59,60]$.

Similarly, the serially concatenated design may be extended to more than two constituent codes $[1,36,42,53]$ as shown in Fig. 3 for a three-stage system. Fig. 3 shows the block diagrams of the encoder and decoder for a three-stage serially concatenated code. As shown in Fig. 3, the data is encoded in three constituent encoders Encoder 1, Encoder 2 and Encoder 3 , invoking the interleavers $\Pi_{1}$ and $\Pi_{2}$ between the encoders. In the decoder of the three-stage serially concatenated codes of Fig. 3, extrinsic information is exchanged between the three constituent decoders in order to attain an improved BER.

\section{A. Interleaver Design}

Interleavers are used between the constituent codes of both serial or parallel concatenated structures in order to reduce the correlations between the data exchanged between the decoders and hence to provide as independent extrinsic information between the constituent decoders as possible [25]. Furthermore, the interleavers are also useful in terms of spreading a burst of errors across the whole decoded frame, hence effectively transforming a bursty channel into a random channel. As a benefit, the errors in a codeword appear to 


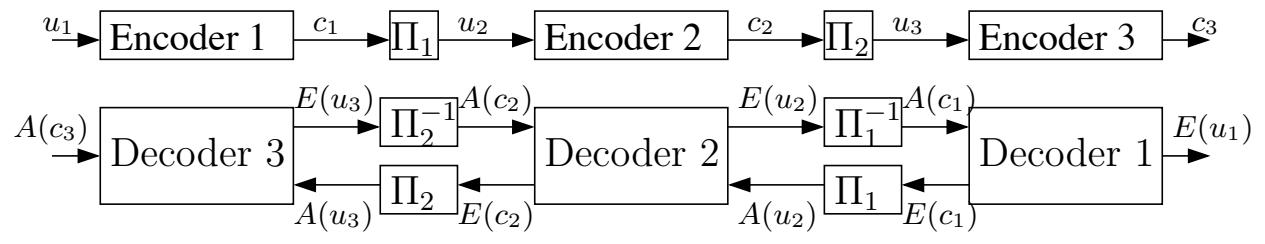

Fig. 3. Encoder and decoder structure of a three-stage serial concatenated code [54].

be independent [61]. Furthermore, using longer interleavers typically leads to more Gaussian-like LLR distributions and hence the system's performance may approach Shannon's capacity limit more closely [25]. The interleaver has been shown to be an essential component of iterative decoding and its length is the dominant factor in determining the code's distance properties, which in turn determines the asymptotic performance of iteratively decoded systems [61-63]. The goal of the interleaver is to improve the distance properties of the code [64]. In summary, the interleaver design substantially affects both the BER "turbo cliff" as well as the error floor.

Several interleaver designs were proposed in [61] for increasing the minimum Hamming distance of parallel concatenated codes. According to [61], at high SNR values, the asymptotic BER of a turbo code decreases linearly upon increasing the interleaver length. The interleaver designs may be categorised as random and deterministic. The random interleavers actually rely on pseudo-random sequences [61]. Several pseudo-random interleavers, such as the S-random interleaver, were described in [65]. On the other hand, the deterministic interleavers are parametrised and hence do not require as large a memory as a random interleaver. Several deterministic interleaver designs were presented in [61].

In contrast to parallel concatenated turbo codes, the serially concatenated codes use interleavers associated with different Hamming distance properties [64]. A theoretical framework conceived for the optimisation of interleavers designed for serially concatenated systems was presented in [66], where the optimisation cost function was related to the asymptotic performance of the system. In [67], an interleaver was proposed for serially concatenated convolutional codes, which was designed for mitigating the correlation between the neighbouring LLRs. This approach increased the minimum Hamming distance and hence reduced the corresponding error floor. Finally, an interleaver algorithm was proposed in [64] for achieving a specific minimum Hamming distance, which is limited only by the degree of freedom offered by the interleaver length. This design was capable of providing a further improved error floor performance compared to the previous designs.

\section{B. Log-likelihood ratios}

The soft information exchanged between the constituent decoders of Fig. 1, Fig. 2 and Fig. 3 is used in the form of Logarithmic-Likelihood Ratios, or LLRs for short. The concept of LLR was proposed by Robertson in [68] in order to simplify the passing of information between the constituent decoders and is now widely used in iterative decoding litera-

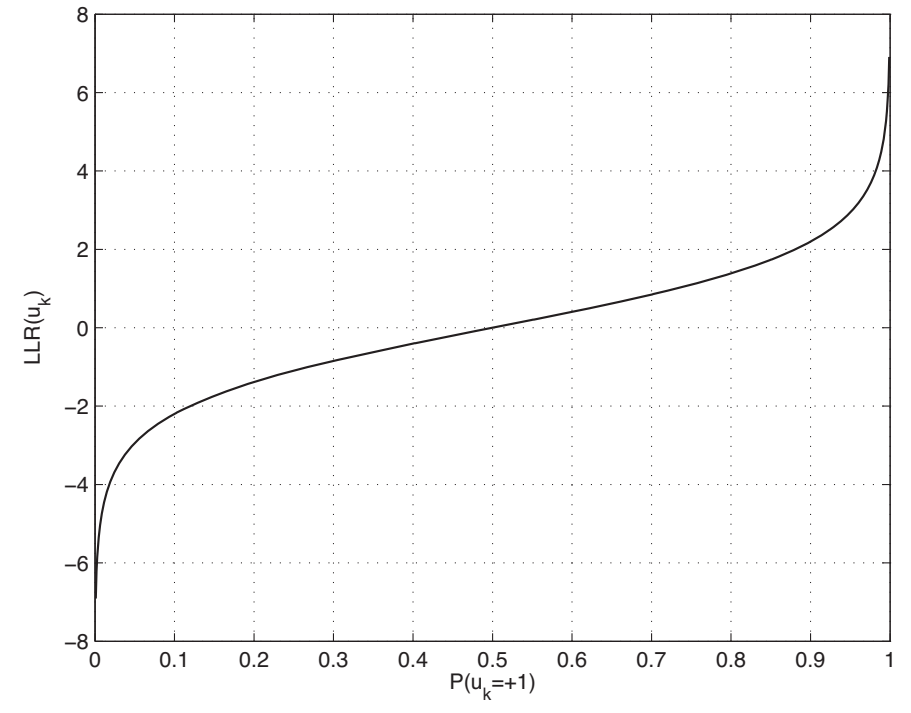

Fig. 4. LLR versus the probability of $u_{k}=+1$.

ture. The LLR of a bit $b_{k}$ is defined as follows:

$$
L\left(b_{k}\right) \triangleq \ln \left(\frac{P\left(u_{k}=+1\right)}{P\left(u_{k}=-1\right)}\right)
$$

where $L\left(b_{k}\right)$ denotes the LLR of bit $b_{k}$ and is defined as the logarithm of the ratios of the probability of the bit $b_{k}$ assuming its two legitimate values. $P\left(u_{k}\right)$ denotes the probability of bit $u_{k}$ having values of +1 and -1 .

Fig. 4 shows how the LLR varies with the probability of $u_{k}=+1$. As seen in Fig. 4, the sign of the LLR indicates whether the bit $u_{k}$ is more likely to be +1 or -1 , while the magnitude of the LLR gives an indication of how likely it is that the sign of the LLR gives the correct value of $u_{k}$. When the LLR $L\left(u_{k}\right) \approx 0$, the probability that the bit $u_{k}=+1$ is equivalent to the probability that the bit $u_{k}=-1$, i.e. $P\left(u_{k}=+1\right)=P\left(u_{k}=-1\right)=0.5$, which indicates that there is no real certainty about the value of $u_{k}$. On the other hand, when $L\left(u_{k}\right) \gg 0$, then $P\left(u_{k}=+1\right) \gg P\left(u_{k}=-1\right)$ and hence it is highly likely that $u_{k}=+1$. Similarly, when $L\left(u_{k}\right) \ll 0$, then $P\left(u_{k}=+1\right) \ll P\left(u_{k}=-1\right)$ and hence it is highly likely that $u_{k}=-1$.

\section{EXIT CHART ANALYSIS}

The main objective of employing EXIT charts proposed by Stephan ten Brink $[24,25]$, is to predict the convergence behaviour of the iterative decoder by examining the evolution of the input/output mutual information exchange between the constituent decoders in consecutive iterations. The application of EXIT charts is based on two assumptions, namely on having a high interleaver length [1], which ensures that 


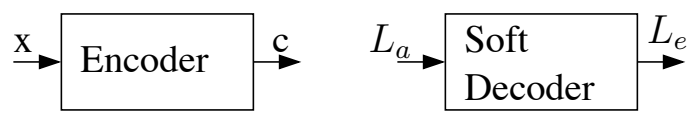

Fig. 5. A block diagram of an encoder and its corresponding soft decoder accepting apriori LLRs and outputting extrinsic LLRs.

- the a priori LLR values are fairly uncorrelated;

- the probability density function of the a priori LLR values is Gaussian.

The EXIT chart analysis may be applied for visualising the convergence behaviour of serially concatenated systems, parallel concatenated systems and hybrid systems combining serial and parallel concatenation. We will continue by highlighting the concept of EXIT charts with the aid of serially concatenated system design examples, noting that the analysis can be extended to parallel concatenated and hybrid systems. In the following, we will describe the concept of mutual information followed by illustrative examples of using EXIT charts for characterising iteratively decoded systems.

\section{A. Mutual information}

The theoretical foundations of information theory are based on Shannon's pioneering work [3], including the concept of Mutual Information (MI) which forms an introduction to the concept of EXIT charts.

Consider an information source generating " $q$ " equiprobable levels, with each level having a probability of $p_{i}=$ $1 / q$, where $i=1, \cdots, q$. The information carried by any of the $q$ levels is defined as $[4,54,69]$

$$
I=\log _{2}\left(\frac{1}{p_{i}}\right)=\log _{2}(q) .
$$

The average information per message emitted by a source is referred to as its entropy, which is defined as $[4,54,69]$ :

$$
H=\sum_{i=1}^{q} p_{i} I_{i}=\sum_{i=1}^{q} p_{i} \log _{2}\left(\frac{1}{p_{i}}\right)=\sum_{i=1}^{q} p_{i} \log _{2}(q) \text { bits. }
$$

When data is transmitted over a non-ideal channel, impairments are introduced and hence the received symbol $Y$ might become different from the transmitted symbol $X$. Denote $P\left(X_{i}\right)$ as the probability that $X_{i}$ was transmitted and $P\left(Y_{j}\right)$ as the probability that $Y_{j}$ was received. Then, $P\left(X_{i}, Y_{j}\right)$ denotes the joint probability that $X_{i}$ was transmitted and $Y_{j}$ was received, while $P\left(X_{i} / Y_{j}\right)$ denotes the conditional probability that $X_{i}$ was transmitted, given that $Y_{j}$ was received. By contrast, $P\left(Y_{j} / X_{i}\right)$ denotes the conditional probability that $Y_{j}$ was received, given that $X_{i}$ was transmitted [4,54]. The MI of $X_{i}$ and $Y_{j}$ is defined as:

$$
\begin{aligned}
I\left(X_{i}, Y_{j}\right) & =\log _{2}\left(\frac{P\left(X_{i} / Y_{j}\right)}{P\left(X_{i}\right)}\right) \\
& =\log _{2}\left(\frac{P\left(Y_{j} / X_{i}\right)}{P\left(Y_{j}\right)}\right) \text { bits. }
\end{aligned}
$$

The average MI, which gives the average amount of source

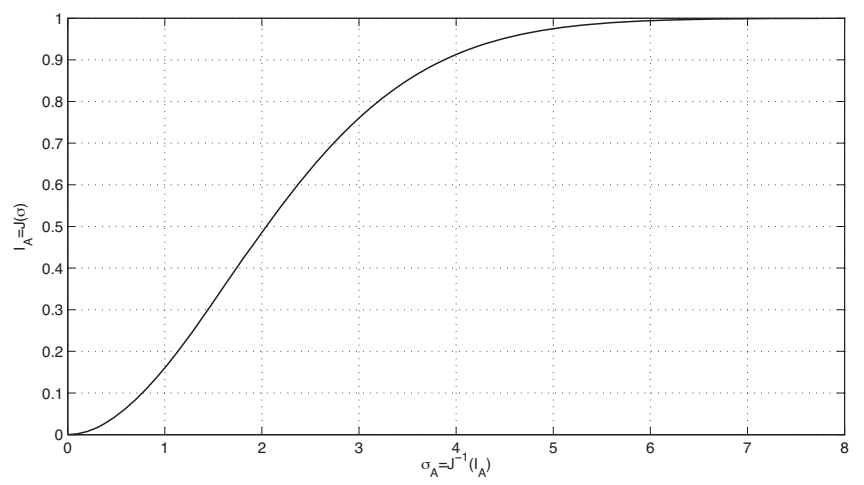

Fig. 6. The MI $I_{A}=J(\sigma)$ as a function of $\sigma_{A}$ evaluated using Equation (10).

information acquired per received symbol, can be defined as:

$$
\begin{aligned}
I(X, Y) & =\sum_{x, y} P\left(X_{i}, Y_{j}\right) \cdot I\left(X_{i}, Y_{j}\right) \\
& =\sum_{x, y} P\left(X_{i}, Y_{j}\right) \cdot \log _{2}\left(\frac{P\left(X_{i} / Y_{j}\right)}{P\left(X_{i}\right)}\right) \text { bits/symbol. }
\end{aligned}
$$

Consider the encoder and its corresponding Soft-Input SoftOutput (SISO) decoder shown in Fig. 5. The encoder encodes the bit stream $\mathrm{x}$ and outputs the encoded bit stream c. On the other hand, the decoder's input is comprised of the $a$ priori LLRs denoted by $L_{a}$ and its output extrinsic LLRs are denoted by $L_{e}$. The MI between the information bit stream $\mathbf{x}$ and the decoder's a priori LLRs $L_{a}$ may be formulated as $I_{A}=I\left(x ; L_{a}\right), 0 \leq I_{A} \leq 1$, and is used for quantifying the information content of the a priori knowledge [70]:

$$
\begin{aligned}
I\left(x, L_{a}\right)= & I_{A} \\
= & \frac{1}{2} \cdot \sum_{x=-1,+1} \int_{-\infty}^{+\infty} p_{A}(\zeta \mid X=x) \\
& \cdot \log _{2} \frac{2 \cdot p_{A}(\zeta \mid X=x)}{p_{A}(\zeta \mid X=-1)+p_{A}(\zeta \mid X=+1)} \mathrm{d} \zeta,
\end{aligned}
$$

where $p_{A}(\zeta \mid X=x)$ is the conditional PDF associated with the a priori LLR $L_{a}$ and the information bits $x$ are assumed to be equiprobable, i.e. we have $P(x=+1)=P(x=-1)=$ $1 / 2$.

In order to compute the MI $I_{A}$ of Equation (7), the conditional PDF $p_{A}(\zeta \mid X=x)$ of the LLR $L_{a}$ has to be known. If we model the a priori LLR $L_{a}$ as an independent zero-mean Gaussian random variable $n_{A}$ having a variance of $\sigma_{A}^{2}$, in conjunction with the encoder's input bits $b \in\{0,1\}$ or equivalently $x \in\{-1,+1\}$ as shown in Fig. 5, the a priori input $L_{a}$ may be written as [24]

$$
L_{a}=\mu_{A} \cdot x+n_{A},
$$

where we have $\mu_{A}=\sigma_{A}^{2} / 2$, since $L_{a}$ is an LLR-value obeying the Gaussian distribution provided that the interleaver used in the system is sufficiently long [71]. Accordingly, the conditional probability density function of the a priori input $L_{a}$ obeys:

$$
p_{A}(\zeta \mid X=x)=\frac{1}{\sqrt{2 \pi} \sigma_{A}} \exp \left(-\frac{\left(\zeta-\frac{\sigma_{A}^{2}}{2} \cdot x\right)^{2}}{2 \sigma_{A}^{2}}\right) .
$$




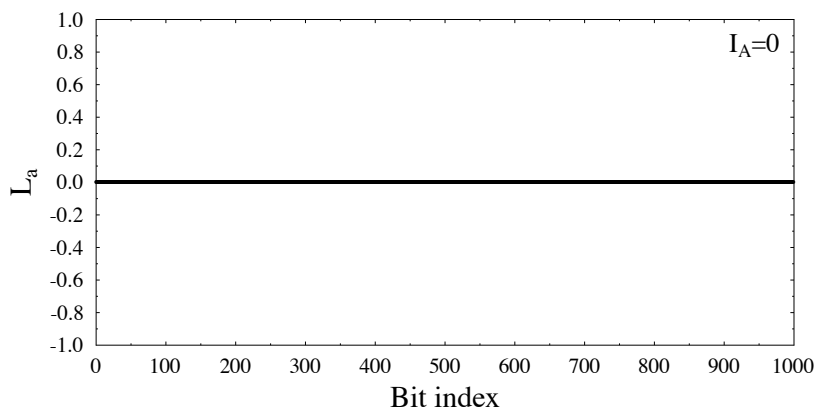

Fig. 7. Soft output from the soft decoder of Fig. 5 for a transmitted stream of $x=+1$ and a mutual information $I_{A}=0$.

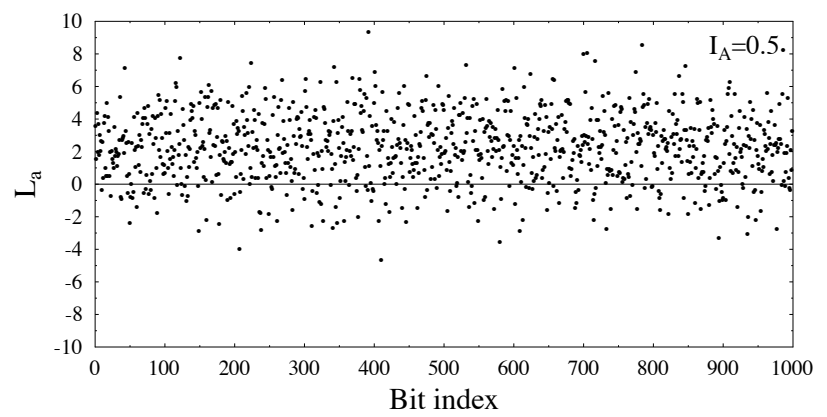

Fig. 8. Soft output from the soft decoder of Fig. 5 for a transmitted stream of $x=+1$ and a mutual information $I_{A}=0.5$.

Consequently, the MI $I_{A}$ given in Equation (7) may alternatively be expressed as:

$$
\begin{aligned}
I_{A}\left(\sigma_{A}\right)= & 1-\frac{1}{\sqrt{2 \pi} \sigma_{A}} \int_{-\infty}^{+\infty} \exp \left(-\frac{\left(\zeta-\frac{\sigma_{A}^{2}}{2}\right)^{2}}{2 \sigma_{A}^{2}}\right) \\
& \log _{2}\left[1+e^{-\zeta}\right] \mathrm{d} \zeta .
\end{aligned}
$$

For notational simplicity and in order to highlight the explicit dependence of $I_{A}$ on $\sigma_{A}$, the following abbreviation is introduced $[24,25]$

$$
J(\sigma):=I_{A}\left(\sigma_{A}=\sigma\right),
$$

where the function $J(\sigma)$ is monotonically increasing and therefore its inverse exists. Additionally, the $J(\sigma)$ function is characterised by $\lim _{\sigma \leftarrow 0} J(\sigma)=0, \lim _{\sigma \leftarrow \infty} J(\sigma)=1, \sigma>0$. Fig. 6 shows a plot of $J(\sigma)$ as a function of $\sigma$.

It was shown in [35] that the MI between the equiprobable bits $X$ and their respective LLRs $L$ for symmetric and consistent ${ }^{1} L$-values always obeys:

$$
\begin{aligned}
I(X ; L) & =1-\int_{-\infty}^{+\infty} p(L \mid X=+1) \cdot \log _{2}\left[1+e^{-L}\right] \mathrm{d} L \\
& =1-E_{X=+1}\left\{\log _{2}\left[1+e^{-L}\right]\right\}
\end{aligned}
$$

where $E_{X}$ is the expectation value of $X$.

For the sake of further illustrating the relation between the LLR and the corresponding MI, Figs. 7-10 show how the

\footnotetext{
${ }^{1}$ The LLR values obeying a symmetric distribution of $p(-\zeta \mid X=+1)=$ $p(\zeta \mid X=-1)$ are said to satisfy the consistency condition [35] iff $p(-\zeta \mid X=$ $x)=e^{-x \zeta} p(\zeta \mid X=x)$ holds.
}

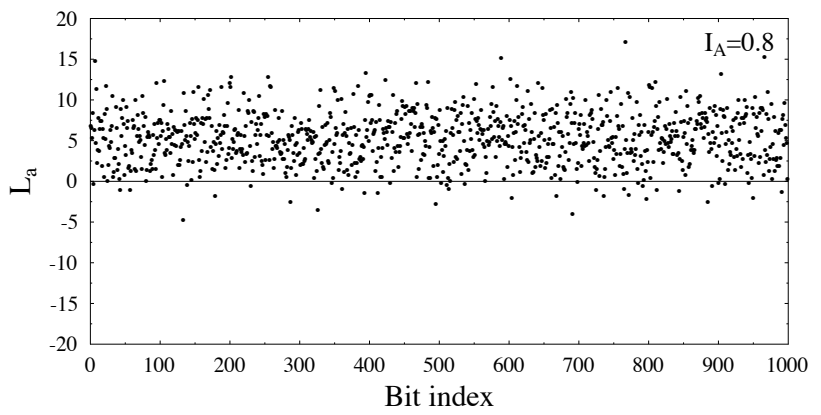

Fig. 9. Soft output from the soft decoder of Fig. 5 for a transmitted stream of $x=+1$ and a mutual information $I_{A}=0.8$.

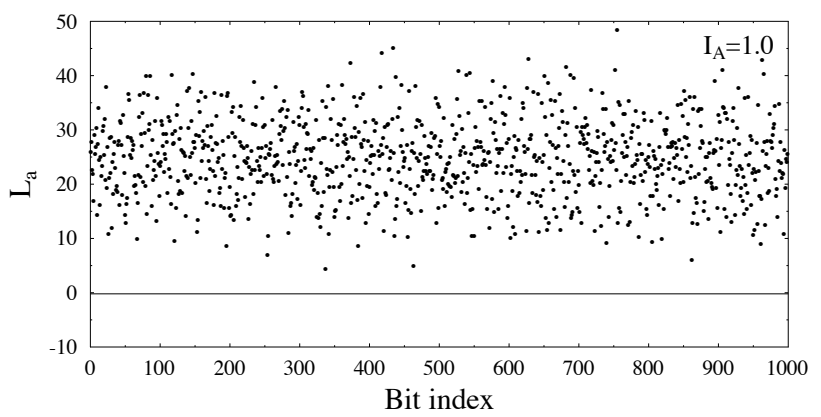

Fig. 10. Soft output from the soft decoder of Fig. 5 for a transmitted stream of $x=+1$ and a mutual information $I_{A}=1.0$.

LLRs $L_{a}$ vary with the corresponding MI values. The bit sequence in this case consists entirely of logical ' 1 's, which is equivalent to $x=+1$, implying that a positive LLR value $L_{a}$ corresponds to a correct hard decision, while a negative one corresponds to an incorrect hard decision. As shown in Fig. 7, an all-zeros LLR sequence corresponds to a MI of 0 , indicating a low confidence that the transmitted bits are 1 or 0 . More explicitly, the probabilities that a 1 or 0 is transmitted are equal. It may be seen from Figs. 7-10 that as the number of negative LLR values decreases, i.e. more LLRs become positive, and the LLR magnitude increases, the corresponding MI increases. By contrast, when the LLR magnitudes are high positive numbers, the MI approaches 1, as shown in Fig. 10. Conversely, when the MI is low, i.e. close to 0 , the corresponding LLRs will have a low magnitude and the wrong sign. As seen in Figs. 7-10, when the MI value increases, the number of LLRs with the incorrect sign is reduced and the magnitude of the correct LLRs is increased until all the LLRs reach a high magnitude with the correct polarity, corresponding to a MI of 1 .

\section{B. EXIT chart analysis of a two-stage serially concatenated system}

The block diagram of a two-stage serially concatenated system is shown in Fig. 11, where the transmitted source bit stream $\mathbf{b}$ is convolutionally encoded by a $1 / 2$-rate Recursive Systematic Convolutional (RSC) code generating the bit stream $\mathbf{c}$, which is then interleaved by a random bit interleaver $\Pi$. The bit interleaved sequence $\mathbf{u}$ is then mapped to the 


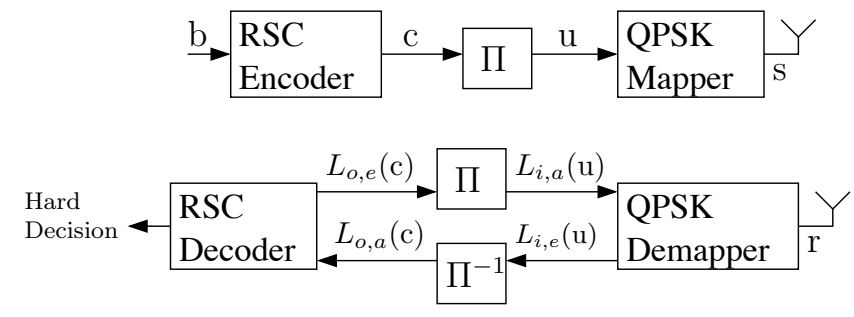

Fig. 11. Block diagram of a two-stage iteratively decoded system using QPSK modulation and RSC code.

symbol-stream $\mathbf{s}$ in the QPSK mapper. The resultant set of symbols may then be transmitted through the channel. We consider a temporally correlated narrowband Rayleigh fading channel, associated with a normalised Doppler frequency of $f_{D}=f_{d} T_{s}=0.01$, where $f_{d}$ is the Doppler frequency and $T_{s}$ is the symbol duration. The complex Additive White Gaussian Noise (AWGN) of $n=n_{I}+j n_{Q}$ contaminates the received signal, where $n_{I}$ and $n_{Q}$ are two independent zero-mean Gaussian random variables having a variance of $\sigma_{n}^{2}=\sigma_{n_{I}}^{2}=\sigma_{n_{Q}}^{2}=N_{0} / 2$ per dimension, with $N_{0} / 2$ representing the double-sided noise power spectral density expressed in $\mathrm{W} / \mathrm{Hz}$.

At the receiver of Fig. 11, the SISO RSC decoder iteratively exchanges extrinsic information with the soft demapper. The RSC decoder invokes the Bahl-Cocke-Jelinek-Raviv (BCJR) algorithm [14] on the basis of a bit-based trellis [72]. All BCJR calculations are performed in the logarithmic probability domain for the sake of converting multiplications to additions and using a lookup table for correcting the Jacobian approximation in the Log Maximum Aposteriori Probability (Log-MAP) algorithm, as discussed in [4, 73, 74].

The extrinsic soft information, represented in the form of the LLRs exmplified in Fig. 4 [71], is iteratively exchanged between the demapper and the RSC decoder of Fig. 11 for the sake of assisting each other's operation, as detailed in $[4,19]$. In Fig. 11, $L(\cdot)$ denotes the LLRs of the bits concerned, where the subscript $i$ indicates the demapper, also often termed as the inner 'decoder', while $o$ corresponds to outer RSC decoder component. Additionally, the subscripts $a$ and $e$ denote the dedicated role of the LLRs with $a$ and $e$ indicating a priori and extrinsic information, respectively.

As shown in Fig. 11, the received complex-valued symbols $\mathbf{r}$ are demapped to their LLR representation for each of the channel-coded bits of a QPSK symbol. The demapper's extrinsic output LLRs $L_{i, e}(\mathbf{u})$ are deinterleaved by a soft-bit deinterleaver, as seen in Fig. 11 to generate the soft bits $L_{o, a}(\mathbf{c})$, which are passed to the RSC decoder as apriori LLRs in order to compute the extrinsic LLR values $L_{o, e}(\mathbf{c})$ provided by the Log-MAP algorithm [4,73] for all the channel-coded bits. During the last iteration, only the LLR values of the original uncoded systematic information bits $\mathbf{b}$ are required, which are passed to the hard decision decoder of Fig. 11 in order to determine the estimated transmitted source bits. As seen in Fig. 11, the extrinsic information generated by the $\mathrm{RSC}$ decoder is then fed back to the demapper as the a priori information $L_{i, a}(\mathbf{u})$ after appropriately reordering them using the interleaver of Fig. 11. The demapper of Fig. 11 exploits

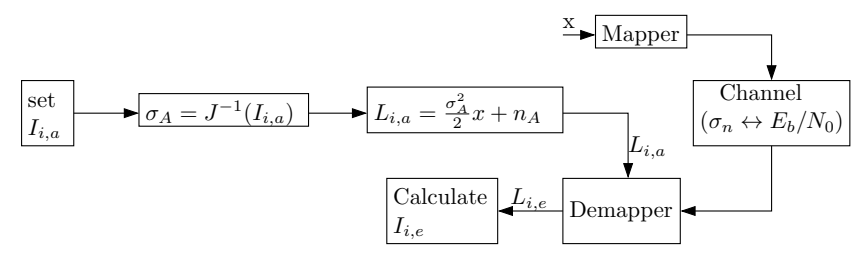

Fig. 12. Evaluation of the demapper transfer characteristic.

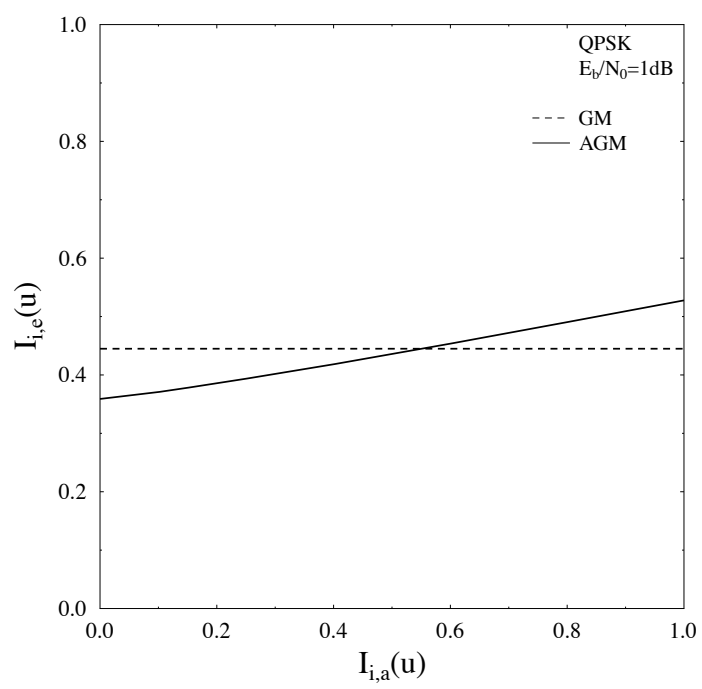

Fig. 13. Symbol-to-bit demapper EXIT characteristics for different bit-tosymbol mapping for the system shown in Fig. 11 operating at $E_{b} / N_{0}=1.0 \mathrm{~dB}$. The number of possible AGM bit-to-symbol mapping schemes depends on the specific modulation scheme considered and here the one having the steepest gradient was provided.

the a priori information for the sake of providing improved extrinsic LLR values, which are then passed to the channel decoder and then back to the demapper for further iterations.

1) Transfer characteristics of the inner demapper: In order to quantify the information content of the extrinsic LLR values $L_{i, e}(\mathbf{u})$ at the output of the demapper, the mutual information $I_{i, e}(\mathbf{u})=I\left[U ; L_{i, e}(\mathbf{u})\right]$ can be used. As seen in Equation (7) in Section III-A using the PDF $p_{E}$ of the extrinsic output LLR $L_{i, e}(\mathbf{u}), I_{i, e}(\mathbf{u})$ can be expressed as [70]:

$$
\begin{aligned}
I_{i, e}(\mathbf{u}) & =\frac{1}{2} \cdot \sum_{x=-1,+1} \int_{-\infty}^{+\infty} p_{E}(\zeta \mid U=u) \\
& \cdot \quad \log _{2} \frac{2 \cdot p_{E}(\zeta \mid U=u)}{p_{E}(\zeta \mid U=-1)+p_{E}(\zeta \mid U=+1)} \mathrm{d} \zeta,
\end{aligned}
$$

or in terms of the function $J($.$) as$

$$
I_{i, e}(\mathbf{u})=J\left(\sigma_{i, e}\right),
$$

where $\sigma_{i, e}$ is the standard deviation of the extrinsic LLRs of the inner decoder.

Observe in Fig. 11 that $I_{i, e}(\mathbf{u})$ is a function of both $I_{i, a}(\mathbf{u})$ and the $E_{b} / N_{0}$ value encountered, hence the demapper's extrinsic information transfer characteristic is defined as [25, $26,46]$

$$
I_{i, e}(\mathbf{u})=T_{i}\left[I_{i, a}(\mathbf{u}), E_{b} / N_{0}\right] .
$$

Fig. 12 illustrates how the EXIT characteristic $T_{i}\left[I_{i, a}(\mathbf{u}), E_{b} / N_{0}\right] \quad$ is calculated for a specific 


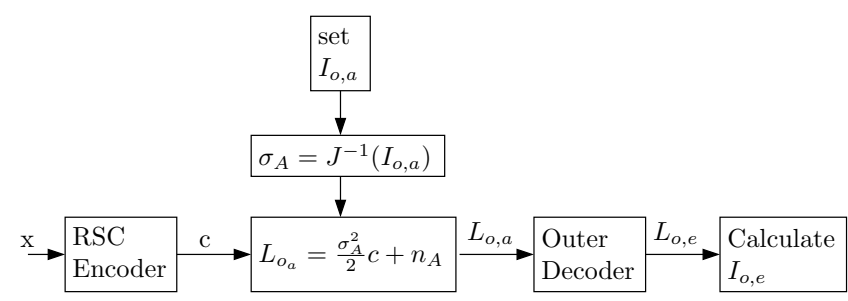

Fig. 14. Evaluation of the RSC decoder's EXIT characteristics.

$\left[I_{i, a}(\mathbf{u}), E_{b} / N_{0}\right]$ input combination. First the noise variance $\sigma_{n}$ of the wireless channel is set according to the $E_{b} / N_{0}$ value considered. Then, $\sigma_{A}$ is calculated based on that specific value of $I_{i, a}(\mathbf{u})$, where the EXIT curve has to be evaluated using the inverse function $\sigma_{A}=J^{-1}\left(I_{i, a}(\mathbf{u})\right)$ expressed from Equation (10) and plotted in Fig. 6. Next, $\sigma_{A}$ is used for creating $L_{i, a}(\mathbf{u})$ according to Equation (7), which is applied as the a priori input of the demapper. Finally, the mutual information of $I_{i, e}(\mathbf{u})=I\left[U ; L_{i, e}(\mathbf{u})\right], 0 \leq I_{i, e}(\mathbf{u}) \leq 1$, between the outer coded as well as interleaved bits $\mathbf{u}$ and the LLR values $L_{i, e}(\mathbf{u})$ is calculated using Equation (13) with the aid of the PDF $p_{E}$ of the extrinsic output $L_{i, e}(\mathbf{u})$. This requires the determination of the distribution $p_{E}$ by means of Monte Carlo simulations. However, according to [35], by invoking the ergodicity theorem of Equation (11), namely by replacing the ensemble average by the time average, the MI can be estimated using a sufficiently large number of samples even for non-Gaussian or unknown distributions, which can be formulated as [35]:

$$
\begin{aligned}
I_{i, e}(\mathbf{u}) & =I\left[X ; L_{i, e}(\mathbf{u})\right] \\
& =1-E_{X=+1}\left\{\log _{2}\left[1+e^{-L_{i, e}}\right]\right\} \\
& \approx 1-\frac{1}{N} \sum_{n=1}^{N} \log _{2}\left[1+e^{-x(n) \cdot L_{i, e}(n)}\right] .
\end{aligned}
$$

Fig. 13 shows the extrinsic information transfer characteristics of the QPSK demapper in conjunction with different bit-to-symbol mapping schemes. As shown in Fig. 13, Gray Mapping (GM) does not provide any iteration gain upon increasing the MI at the input of the demapper. However, using the multi-dimensional hupercube labelling defined for QPSK in $[75,76]$, which we refer to as Anti-Gray Mapping (AGM) ${ }^{2}$, results in different EXIT characteristics, as illustrated by the continuous line seen in Fig. 13.

2) Transfer characteristics of the RSC decoder: The EXIT characteristic of the RSC decoder of Fig. 11 describes the relationship between the RSC apriori input $L_{o, a}(\mathbf{c})$ and the RSC extrinsic output $L_{o, e}(\mathbf{c})$. The RSC decoder has a single input namely the a priori input $L_{o, a}(\mathbf{c})$ provided by the symbol-to-bit demapper. Therefore, the EXIT characteristics of the outer channel decoder is independent of the $E_{b} / N_{0}$ value and hence may be written as

$$
I_{o, e}(\mathbf{c})=T_{o}\left[I_{o, a}(\mathbf{c})\right],
$$

where $I_{o, a}(\mathbf{c})=I\left[C ; L_{o, a}(\mathbf{c})\right], 0 \leq I_{o, a}(\mathbf{c}) \leq 1$, is the MI between the RSC coded bits $\mathrm{c}$ and the apriori LLR

\footnotetext{
${ }^{2}$ Any bit-to-symbol mapping, which is not a Gray mapping, is termed as AGM.
}

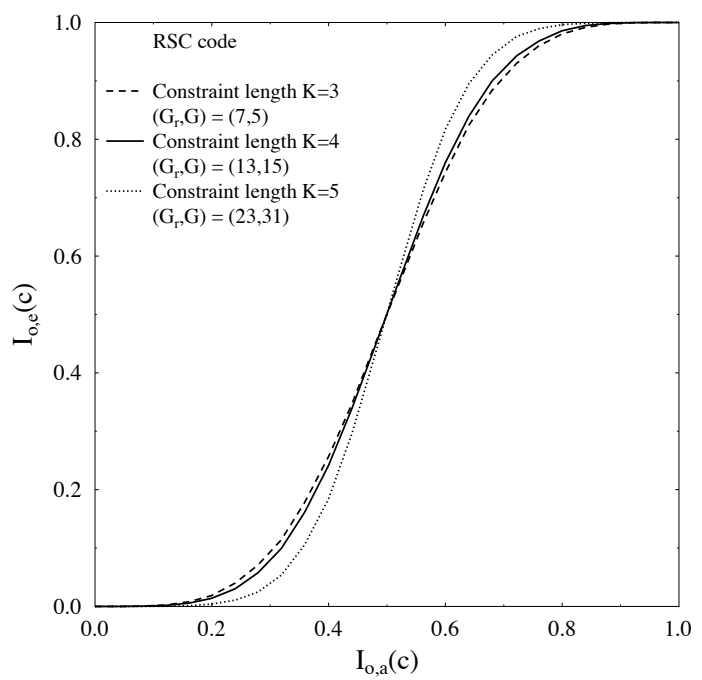

Fig. 15. Extrinsic information transfer characteristics of 1/2-rate RSC codes having different constraint lengths.

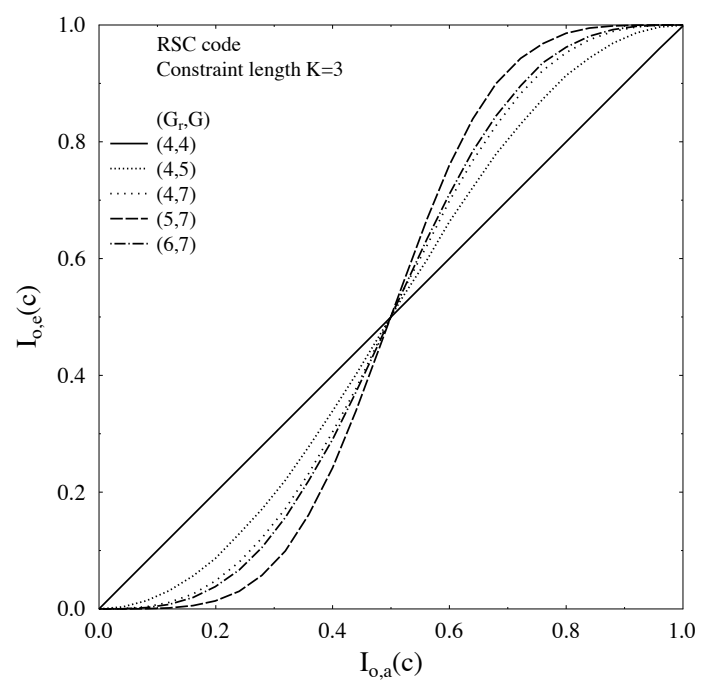

Fig. 16. Extrinsic information transfer characteristics of $1 / 2$-rate RSC codes having a constraint length of $K=3$ and different generator polynomials.

values $L_{o, a}(\mathbf{c})$ and similarly, $I_{o, e}(\mathbf{c})=I\left[C ; L_{o, e}(\mathbf{c})\right], 0 \leq$ $I_{o, e}(\mathbf{c}) \leq 1$, is the MI between the RSC coded bits $\mathbf{c}$ and the extrinsic LLR values $L_{o, e}(\mathbf{c})$. The computational model of evaluating the EXIT characteristics of the outer channel decoder is shown in Fig. 14. As seen from the figure, the procedure is similar to that of the demapper shown in Fig. 12, except that its value is independent of the SNR. Again, $I_{o, e}(\mathbf{c})=I\left[C ; L_{o, e}(\mathbf{c})\right]$ can be computed either by evaluating the histogram based approximation of $p_{E}[25,26,46]$ and then applying Equation (13) or, more conveniently, by the time averaging method [35] of Equation (15) as

$$
\begin{aligned}
I_{o, e}(\mathbf{c}) & =I\left[C ; L_{o, e}(\mathbf{c})\right] \\
& =1-E\left\{\log _{2}\left[1+e^{-L_{o, e}}\right]\right\} \\
& \approx 1-\frac{1}{N} \sum_{n=1}^{N} \log _{2}\left[1+e^{-c(n) \cdot L_{o, e}(n)}\right] .
\end{aligned}
$$




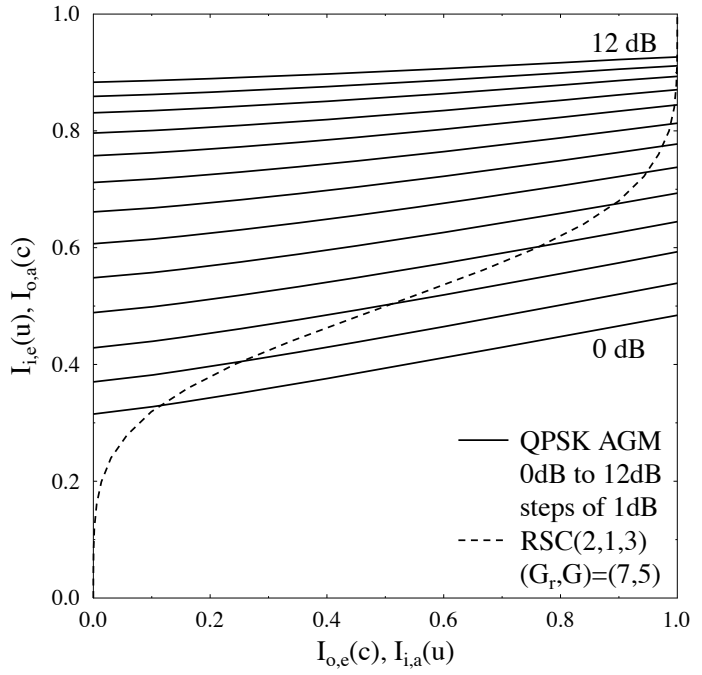

Fig. 17. EXIT chart of an iteratively detected RSC-coded QPSK scheme of Fig. 11 in combination with the outer $\operatorname{RSC}(2,1,3)$ code using the GP of $\left(G_{r}, G\right)=(7,5)$, while communicating over a temporally correlated Rayleigh fading channel exhibiting a normalised Doppler frequency of $f_{D}=0.01$.

The EXIT characteristics of various $1 / 2$-rate RSC codes having different constraint lengths $K$ are shown in Fig. 15. The Generator Polynomials (GP) employed are given in the figure's legend in octal form, where $G_{r}$ is the feedback polynomial and $G$ is the feed-forward polynomial. Fig. 15 demonstrates that for $I_{o, a}(\mathbf{c})>0.5$, the set of RSC codes having higher constraint lengths converge faster upon increasing $I_{o, a}(\mathbf{c})$ than the RSC codes having smaller constraint lengths. Furthermore, observe in Fig. 15 that the extrinsic characteristics of the RSC codes having constraint lengths of $K=3$ and $K=4$ are close to each other. However, the specific shape of the two extrinsic MI curves is more dependent on the generator polynomial used, as shown in Fig. 16. Explicitly, the extrinsic transfer characteristics of several $1 / 2$-rate RSC codes having a constraint length of $K=3$ and diverse GPs are shown in Fig. 16, where the code using the GPs $\left(G_{r}, G\right)=(5,7)$ converges faster than the other codes.

3) Extrinsic information transfer charts: The exchange of extrinsic information in the system of Fig. 11 can be visualised by plotting the EXIT characteristics of the inner demapper and of the outer RSC decoder in an EXIT chart [24, 25]. The outer RSC decoder's extrinsic output information $I_{o, e}(\mathbf{c})$ becomes the demapper's a priori input information $I_{i, a}(\mathbf{u})$, which is represented on the $x$-axis of the EXIT chart. Similarly, on the $y$-axis we plot the demapper's extrinsic output information $I_{i, e}(\mathbf{u})$, which becomes the outer RSC decoder's a priori input information $I_{o, a}(\mathbf{c})$. The EXIT curves presented in this section correspond to the system employing a 1/2-rate RSC code having a constraint length of $K=3$, denoted as $\operatorname{RSC}(2,1,3)$, in conjunction with an octal GP of $\left(G_{r}, G\right)=(7,5)$.

Fig. 17 shows the EXIT chart of the iteratively detected RSC-coded system of Fig. 11 employing QPSK relying on the AGM $[75,76]$ of Fig. 13. Ideally, in order for the exchange of extrinsic information between the demapper and the outer RSC

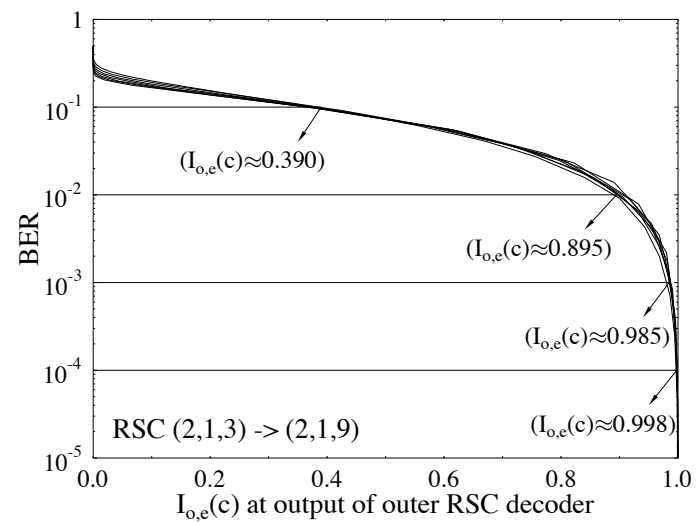

Fig. 18. BER of different 1/2-rate RSC decoders using the maximum-freedistance GPs versus their extrinsic output $I_{E_{D}}$, when increasing the code's constraint length from $K=3$ to $K=9$.

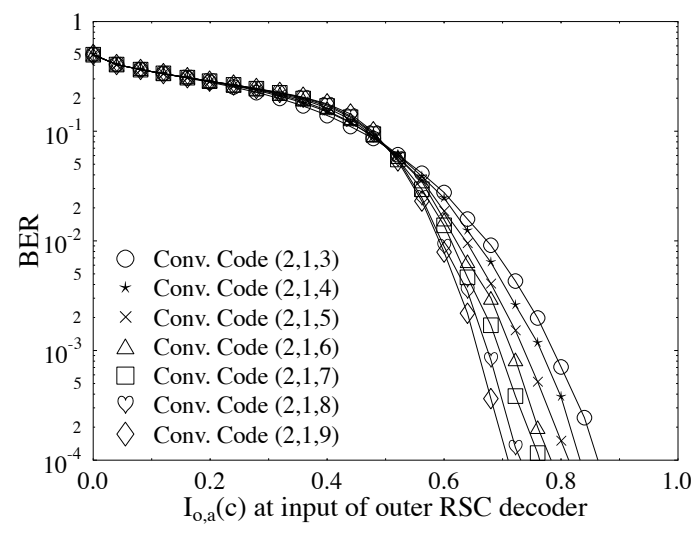

Fig. 19. BER of different 1/2-rate RSC decoders using the maximum-freedistance GPs versus their a priori input $I_{A_{D}}$.

decoder to converge for the sake of achieving a vanishingly low BER at a specific $E_{b} / N_{0}$ value, the EXIT curve of the demapper at the $E_{b} / N_{0}$ value of interest and the extrinsic MI transfer curve of the outer RSC decoder should not intersect before reaching the $(1.0,1.0)$ point. This implies that given $I_{a}=1.0$, we have $I_{e}=1.0$ and provided that this condition is satisfied, a so-called open-convergence tunnel [24,25] appears in the EXIT chart. The narrower the tunnel, the more iterations are required for reaching the $(1.0,1.0)$ point and the closer the BER curve approaches the vertical line representing the channel capacity. If however, the two extrinsic MI transfer characteristics intersect at a point close to the vertical line at $I_{i, a}(\mathbf{u})=1.0$ rather than at the $(1.0,1.0)$ point, then a low BER may still be achieved, although it will remain higher than the BER of the schemes having an intersection at the $(1.0,1.0)$ point. These types of EXIT tunnels are referred to here as semi-convergent tunnels. Observe in Fig. 17 that a semi-convergent tunnel exists at around $E_{b} / N_{0}=8.0 \mathrm{~dB}$. This implies that according to the predictions of the EXIT chart seen in Fig. 17, the iterative decoding process is expected to converge at an $E_{b} / N_{0}$ value between $8.0 \mathrm{~dB}$ and $9.0 \mathrm{~dB}$. These EXIT chart based decoding convergence predictions are usually verified by the Monte-Carlo simulation based iterative decoding trajectory, as will be discussed in Section III-B4.

Observe in the EXIT chart of Fig. 17 that once a semi- 
TABLE II

$I_{o, a}(\mathbf{c})$ REQUIRED AT THE INPUT OF THE RSC DECODER FOR ACHIEVING A BER OF $10^{-4}$.

\begin{tabular}{|c|c|}
\hline Constraint length & Required $I_{o, a}(\mathbf{c})$ \\
\hline 3 & 0.863 \\
\hline 4 & 0.832 \\
\hline 5 & 0.813 \\
\hline 6 & 0.783 \\
\hline 7 & 0.764 \\
\hline 8 & 0.731 \\
\hline 9 & 0.710 \\
\hline
\end{tabular}

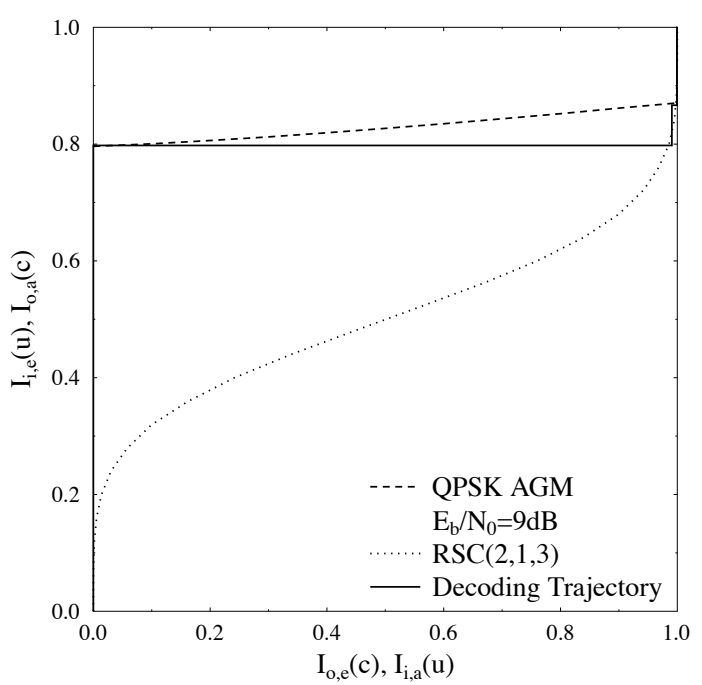

Fig. 20. Monte-Carlo simulation based decoding trajectory of the iteratively detected system of Fig. 11 in conjunction with QPSK using AGM at $E_{b} / N_{0}=9.0 \mathrm{~dB}$ for an interleaver length of 800,000 bits.

convergent tunnel is formed, the intersection point of the extrinsic MI transfer characteristic curves of the inner symbolto-bit demapper and the outer RSC decoder gradually moves towards the $(1.0,1.0)$ point upon increasing the SNR. In order to investigate how the position of the intersection point affects the BER performance, Fig. 18 shows the achievable BER as a function of the mutual information $I_{o, e}(\mathbf{c})$ at the output of the RSC decoder for different constraint lengths. According to Fig. 18, the point of intersection should be at least at $I_{o, e}(\mathbf{c})=0.985$ in order to achieve a BER of $10^{-3}$, which is practically independent of the RSC code's constraint length. This is true, because Fig. 18 relates the MI at the output of the RSC decoder to the achievable BER. Fig. 19, however, relates the MI at the input of the RSC decoder to the achievable BER. The effect of the code's constraint length becomes evident in Fig. 19, since RSC codes having higher constraint lengths require lower $I_{o, a}(\mathbf{c})$ values in order to achieve a similar BER. Table II summarises the $I_{o, a}(\mathbf{c})$ values required for achieving a BER of $10^{-4}$ at the input of the RSC decoder of Fig. 11.

4) Decoding trajectory: As mentioned in Section III-B3, the EXIT chart based convergence predictions can be verified by the Monte-Carlo simulation based iterative decoding trajectory. Fig. 20 illustrates the corresponding decoding trajectory of the iteratively decoded system of Fig. 11 at $E_{b} / N_{0}=9.0 \mathrm{~dB}$ in conjunction with QPSK AGM and using an interleaver

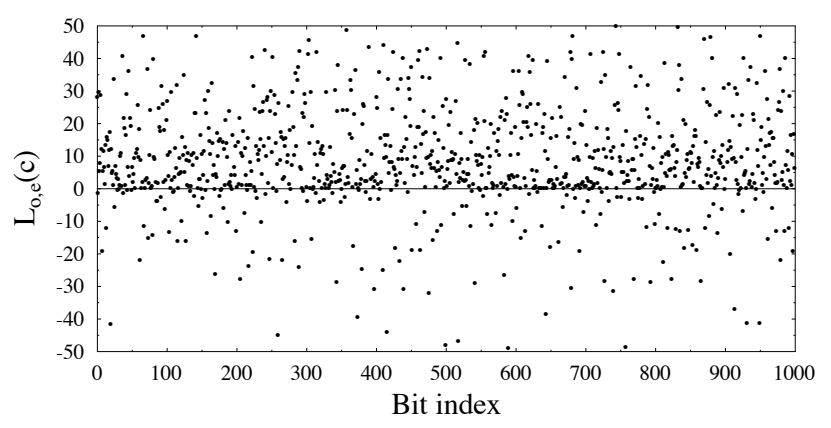

Fig. 21. Soft output of the $1 / 2$-rate, $K=3,\left(G_{r}, G\right)=(5,7)$ RSC decoder of Fig. 11 for a transmitted stream of all +1 during the first iteration at $E_{b} / N_{0}=9.0 \mathrm{~dB}$. The mutual information at the output of the RSC decoder is $I_{o, e}(c)=0.886$.

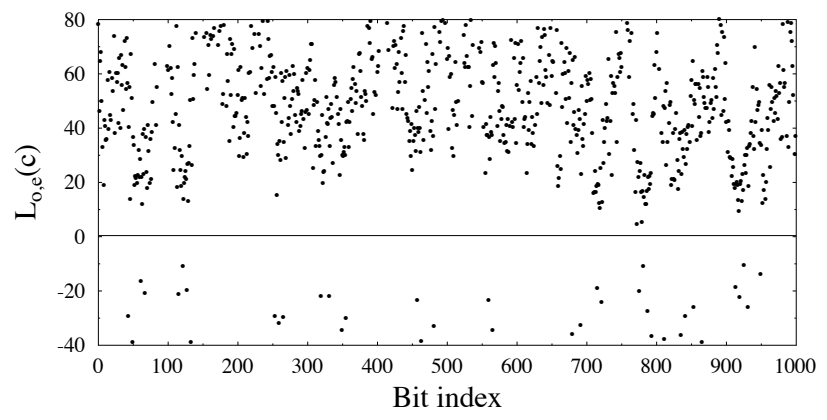

Fig. 22. Soft output of the $1 / 2$-rate, $K=3,\left(G_{r}, G\right)=(5,7)$ RSC decoder of Fig. 11 for a transmitted stream of all +1 during the second iteration at $E_{b} / N_{0}=9.0 \mathrm{~dB}$. The mutual information at the output of the RSC decoder is $I_{o, e}(c)=0.935$. Observe that in contrast to Fig. 21, a substantial reduced number of erroneous bit-decisions would emerge.

depth of 800,000 bits. The horizontal and vertical steps seen in Fig. 20 represent the actual extrinsic information transfer between the inner demapper and the outer RSC channel decoder of Fig. 11.

Figs. 21 and 22 show how the LLRs $L_{o, e}(c)$ are affected by increasing the number of decoding iterations from one to two. The bit sequence in this case consists entirely of logical ' 1 's, which is equivalent to $x=+1$. This implies that a positive LLR value $L_{o, e}(c)$ corresponds to a correct hard decision and a negative LLR value corresponds to an incorrect hard decision. The LLRs shown in Fig. 21 and Fig. 22 are shown for $E_{b} / N_{0}=9.0 \mathrm{~dB}$. As observed in Fig. 21, during the first iteration there are lots of errors and this is manifested by the negative LLRs shown in the figure. The MI for the LLRs in the first iteration is $I_{o, e}(c)=0.886$. By contrast, Fig. 22 shows that during the second iteration the amplitude of the LLRs increases and there are less LLRs with a negative sign. The MI in this case is $I_{o, e}(c)=0.935$. As shown in the decoding trajectory of Fig. 20, there is no further gain after two iterations and the EXIT curves of the inner demapper and the outer RSC decoder intersect before reaching the $(1.0,1.0)$ point. Hence there will be still residual errors in this case and this is clearly indicated by the LLRs with the negative sign in Fig. 22.

The BER of the iteratively decoded system of Fig. 11 is shown in Fig. 23. The figure compares the BER performance of the GM- and AGM-aided QPSK modulated system for a 


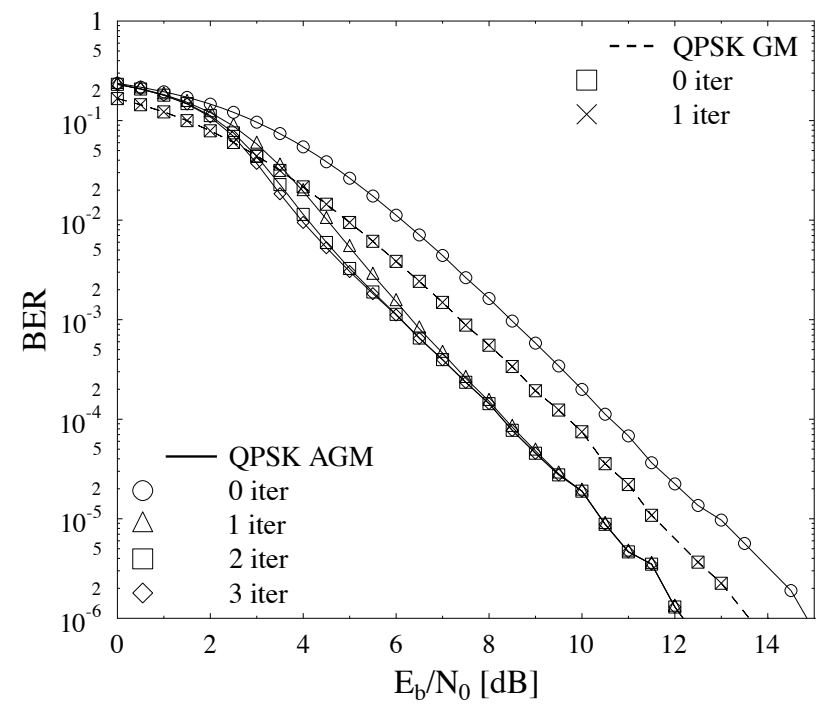

Fig. 23. BER Performance of the AGM- and GM-based iteratively detected RSC-coded system shown in Fig. 11 in conjunction with an interleaver depth of 800,000 bits while using a variable number of iterations.

variable number of iterations, while employing an interleaver depth of 800,000 bits and communicating over a narrowband Rayleigh fading channel having a normalised Doppler frequency of 0.01. As shown in Fig. 23, the BER performance of the GM-aided system does not improve upon increasing the number of iterations and this matches the EXIT chart prediction of Fig. 13, where the GM-based system has a flat EXIT transfer function. On the other hand, the AGM-based system's performance improves upon increasing the number of iterations from 0 to 1 , but beyond that the BER performance no longer improves upon increasing the number of iterations. This trend is confirmed by the decoding trajectory of Fig. 20, where it was shown that the EXIT curves of the inner demapper and the outer RSC decoder intersect after the first iteration. Also note that the BER curves of Fig. 23 do not exhibit a sharply decaying "turbo-cliff" shape that is expected in an iteratively decoded system. This is due to the fact that the EXIT transfer functions of the inner and outer decoders intersect before reaching the $(1.0,1.0)$ point of perfect convergence to an infinitesimally low BER and hence we experience a gradually, rather than sharply, decaying BER curve in Fig. 23.

\section{EXIT chart analysis of a two-stage serially concatenated system using a recursive inner code}

The BER results shown in the previous section did not exhibit a steep turbo-cliff due to the fact that the EXIT curve of the inner demapper and the outer RSC decoder intersected before reaching the $(1.0,1.0)$ point. It was shown in [52] that a recursive inner decoder component is needed in order to maximise the interleaver gain and to avoid the formation of a BER floor, when employing iterative decoding. In [45], unityrate inner codes were employed for designing low-complexity iterative detection aided schemes suitable for bandwidth and power limited systems having stringent BER requirements.
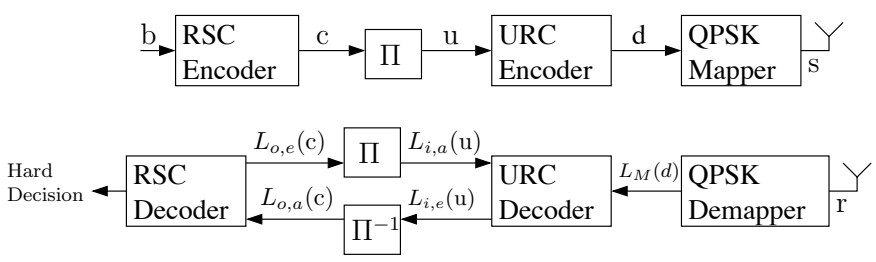

Fig. 24. Block diagram of a two-stage iteratively decoded system using QPSK modulation in conjunction with URC and RSC codes.

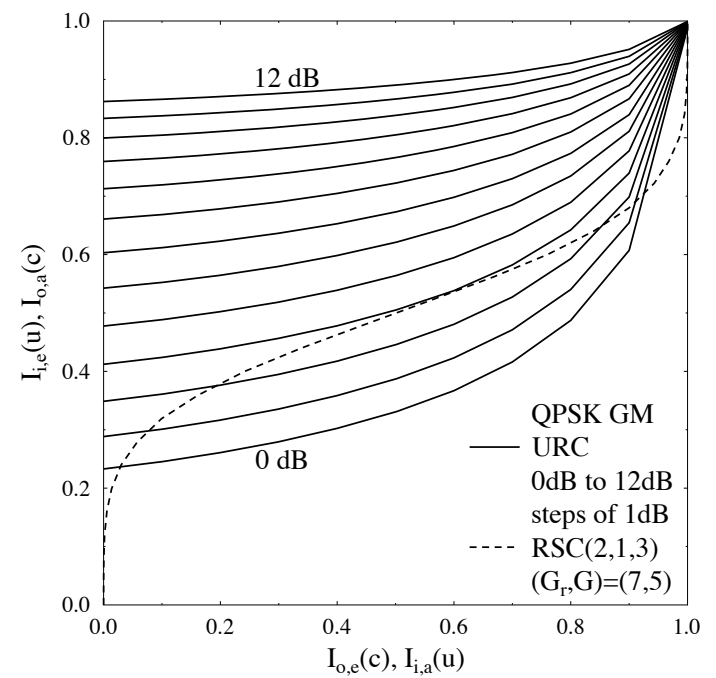

Fig. 25. EXIT chart of the $1 / 2$-rate $K=3$ RSC-coded and URC-precoded scheme of Fig. 24 employing GM QPSK, while using an interleaver depth of 800,000 bits, which should be contrasted to the EXIT chart of Fig. 17 based on the schematic of Fig. 11 using no URC.

Hence, in this section we consider the iteratively decoded system of Fig. 24, where iterative detection is carried out by exchanging extrinsic information between the outer RSC decoder and the inner Unity-Rate Code's (URC) decoder. An explicit benefit of the URC is that it has an Infinite Impulse Response (IIR) and hence it efficiently spreads/randomises the extrinsic information without increasing the interleaver delay and without reducing the throughput. This is achieved at a modest complexity increase, because a memory-1 URC has only two trellis states.

The schematic of the proposed system is shown in Fig. 24, where the transmitted source bits $\mathbf{b}$ are convolutionally encoded by a $1 / 2$-rate memory- $2 K=3$ RSC code having a GP octally represented as $\left(G_{r}, G\right)=(7,5)$. The RSC encoded bit stream $\mathbf{c}$ is then interleaved by a random bit interleaver. Following channel interleaving the bit stream $\mathbf{u}$ is encoded by a URC encoder. More specifically, the URC encoder is a simple rate-1 memory-1 accumulator, which is described by the pair of octal GPs of $\left(G_{r}, G\right)=(3,2)$. The QPSK mapper of Fig. 24 then maps the RSC coded and URC precoded bits to QPSK symbols for transmission from a single antenna. Similarly to Section III-B, we consider transmission over a temporally correlated narrowband Rayleigh fading channel associated with a normalised Doppler frequency of $f_{D}=f_{d} T_{s}=0.01$.

As shown in Fig. 24, the received complex-valued symbols 


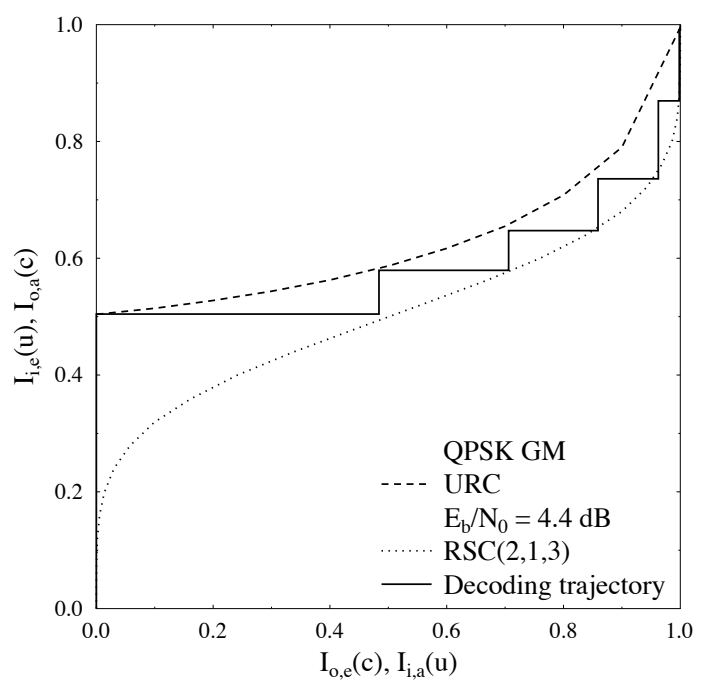

Fig. 26. Decoding trajectory of the $1 / 2$-rate, $K=3$ RSC-coded and URCprecoded scheme of Fig. 24 employing GM QPSK, while using an interleaver depth of 800,000 bits and operating at $E_{b} / N_{0}=4.4 \mathrm{~dB}$.

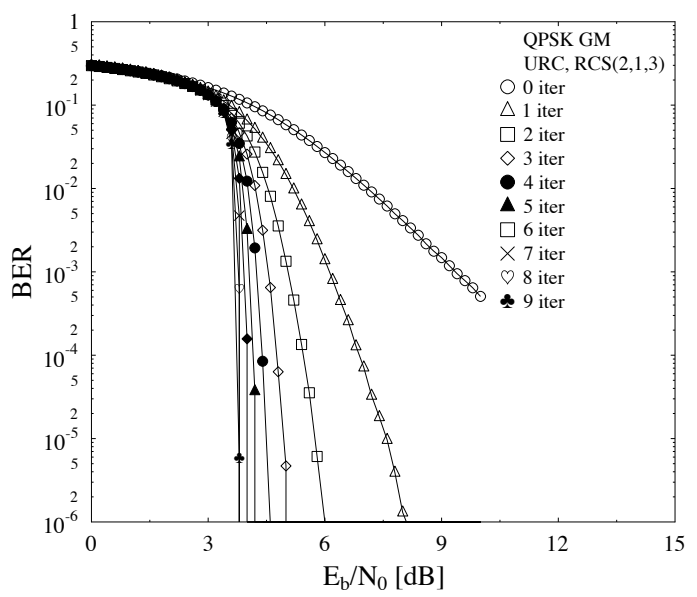

Fig. 27. BER performance of the $1 / 2$-rate, $K=3$ RSC-coded and URCprecoded scheme of Fig. 24 employing GM QPSK, while using an interleaver depth of 800,000 bits. Contrast the significantly improved BER performance to that seen in Fig. 23 for the scheme of Fig. 11 dispensing with URC.

are first demodulated and demapped to the soft values of $L_{M}(\mathbf{d})$, which are then passed to the URC decoder. As seen in Fig. 24, the URC decoder processes the information provided by the demapper in conjunction with the a priori information $L_{i, a}(\mathbf{u})$ passed from the RSC decoder, in order to generate the extrinsic LLRs $L_{i, e}(\mathbf{u})$. Then the LLRs $L_{i, e}(\mathbf{u})$ are deinterleaved by a soft-bit deinterleaver, as seen in Fig. 24. Next, the soft-bit values $L_{o, a}(\mathbf{c})$ are passed to the RSC decoder of Fig. 24 in order to compute the extrinsic LLRs $L_{o, e}(\mathbf{c})$. More iterations are performed by exchanging soft extrinsic information between the RSC and URC decoders. During the last iteration, only the LLR values of the original uncoded systematic information bits are required, which are passed to the hard decision decoder of Fig. 24 in order to determine the estimated transmitted source bits.

Fig. 25 depicts the EXIT chart of the iterative detection aided channel-coded system of Fig. 24 employing GM QPSK

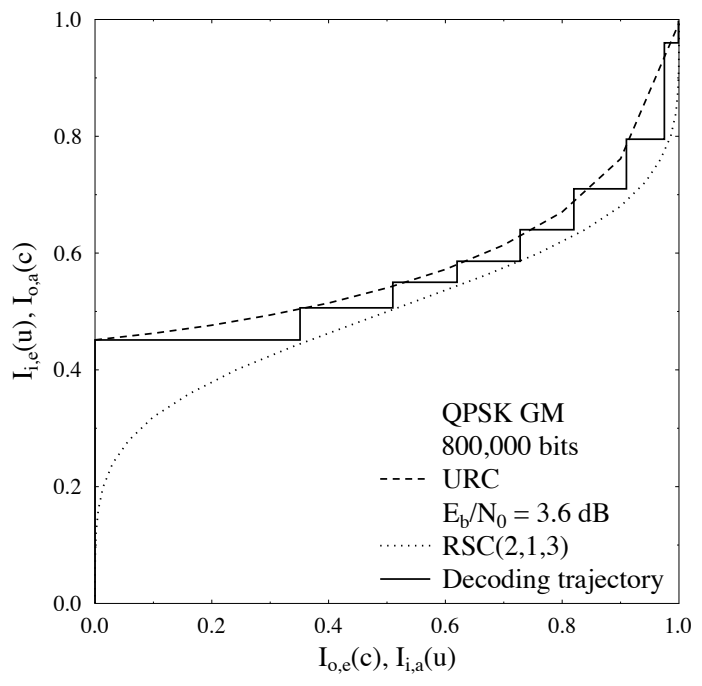

Fig. 28. Decoding trajectory of the $1 / 2$-rate, $K=3$ RSC-coded and URCprecoded scheme of Fig. 24 employing GM QPSK, while using an interleaver depth of 800,000 bits and operating at $E_{b} / N_{0}=3.6 \mathrm{~dB}$. Comparing this figure to Fig. 26 it can be inferred that reaching a given $\mathrm{BER}$ at $3.6 \mathrm{~dB}$ requires almost twice the number of iterations in comparison to $4.4 \mathrm{~dB}$.

in conjunction with the $1 / 2$-rate RSC outer code and URC inner code for different $E_{b} / N_{0}$ values. The classic GM was used in this case, because no iterations are invoked between the demapper and the decoders and thus, in this case, it is better to use GM that results in a higher initial MI and hence at higher starting point at $I_{A}=0$ in the EXIT curve. Ideally, in order for the exchange of extrinsic information between the URC decoder and the RSC decoder to converge at a specific $E_{b} / N_{0}$ value, the EXIT curve of the URC decoder and that of the outer RSC decoder should only intersect at a point near the $I_{o, e}(\mathbf{c})=1.0$ line. It is plausible that the narrower the open EXIT tunnel, the more iterations are required for reaching the vertical $I_{o, e}(\mathbf{c})=1.0$ line. Observe from the figure that an open convergence tunnel is formed at an $E_{b} / N_{0}$ between $3.0 \mathrm{~dB}$ and $4.0 \mathrm{~dB}$. This implies that according to the predictions of the EXIT chart seen in Fig. 25, the iterative decoding process is expected to converge at $E_{b} / N_{0} \in[3.0,4.0] \mathrm{dB}$.

The EXIT chart based convergence predictions may be verified by the Monte-Carlo simulation based iterative decoding trajectory of Fig. 26, where the trajectory at $E_{b} / N_{0}=4.4 \mathrm{~dB}$ is recorded, while using an interleaver depth of 800,000 bits. The steps seen in the figure represent the actual extrinsic MI transfer between the inner URC decoder and the outer RSC decoder. Since a long interleaver is employed, the assumptions outlined at the beginning of Section III namely those of having uncorrelated and Gaussian distributed a priori LLRs, are justified and hence the EXIT chart based convergence prediction becomes accurate. On the other hand, Fig. 28 shows the trajectory of the iteratively decoded system of Fig. 24 at $E_{b} / N_{0}=3.6 \mathrm{~dB}$, while using an interleaver depth of 800,000 bits. Observe in Fig. 28 that the open tunnel recorded at $E_{b} / N_{0}=3.6 \mathrm{~dB}$ is narrower than that at $E_{b} / N_{0}=3.6 \mathrm{~dB}$ as seen in Fig. 26. Hence, as shown in Fig. 26 and Fig. 28, more iterations are needed for reaching the point of perfect convergence at $(1.0,1.0)$ at $3.6 \mathrm{~dB}$ than at $4.4 \mathrm{~dB}$.

Fig. 27 shows the attainable performance of the RSC-coded 


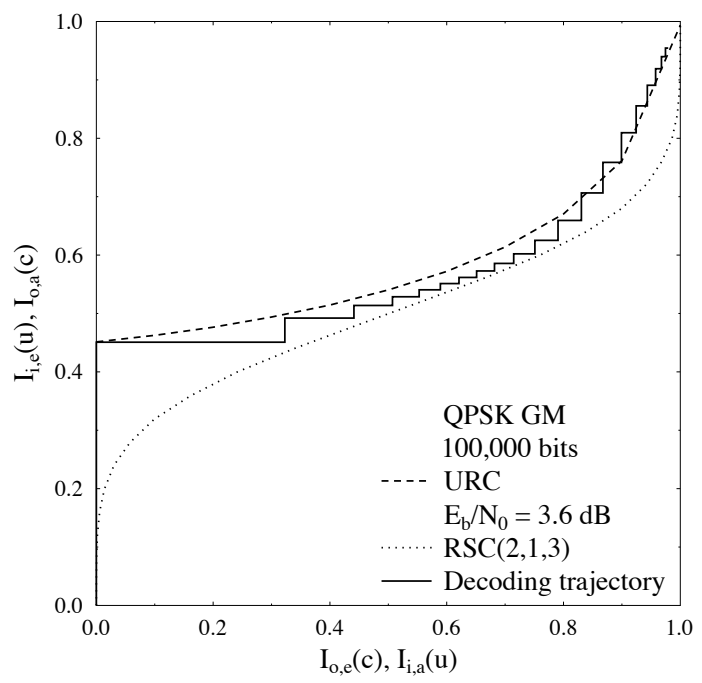

Fig. 29. Decoding trajectory of a $1 / 2$-rate, $K=3$ RSC-coded and URCprecoded scheme of Fig. 24 employing GM QPSK, while using an interleaver depth of 100,000 bits and operating at $E_{b} / N_{0}=3.6 \mathrm{~dB}$.

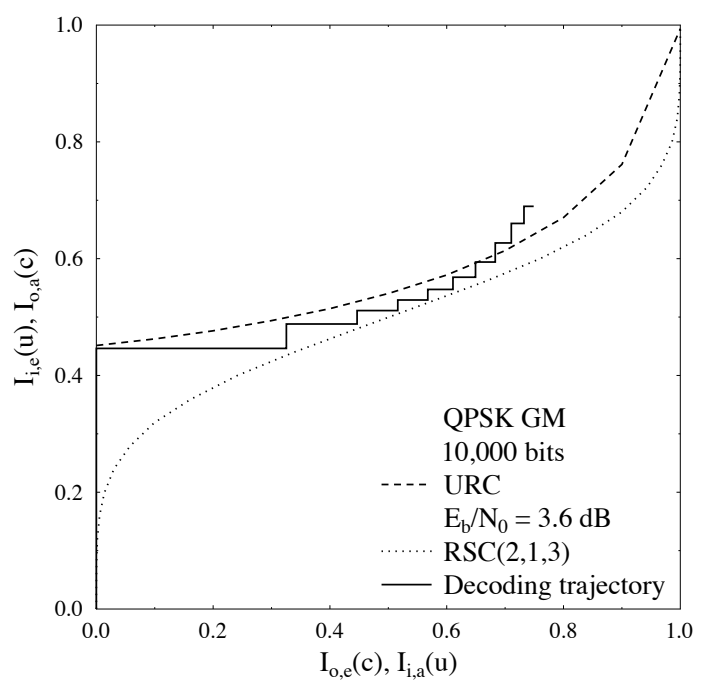

Fig. 30. Decoding trajectory of a $1 / 2$-rate, $K=3$ RSC-coded and URCprecoded scheme of Fig. 24 employing GM QPSK, while using an interleaver depth of 10,000 bits and operating at $E_{b} / N_{0}=3.6 \mathrm{~dB}$.

and URC-precoded system of Fig. 24 employing GM QPSK in conjunction with a $1 / 2$-rate RSC code and an interleaver depth of 800,000 bits, while using a gradually increasing number of decoding iterations between the RSC and URC decoders of Fig. 24. Observe in Fig. 27 that there is an evident performance improvement, when increasing the number of iterations as compared to the modest improvement recoreded for the system of Fig. 11 dispensing with the inner recursive URC encoder. As shown in Fig. 27, the system attains an infinitesimally low BER at around $E_{b} / N_{0}=3.6 \mathrm{~dB}$, which matches with the EXIT chart prediction of Fig. 25. Furthermore, according to Fig. 25 there is an open tunnel between the inner and outer decoders' EXIT curves at $E_{b} / N_{0}$ just above $3.0 \mathrm{~dB}$. This implies that it is possible to obtain an infinitesimally low BER at $E_{b} / N_{0}$ just above $3.0 \mathrm{~dB}$, albeit this requires

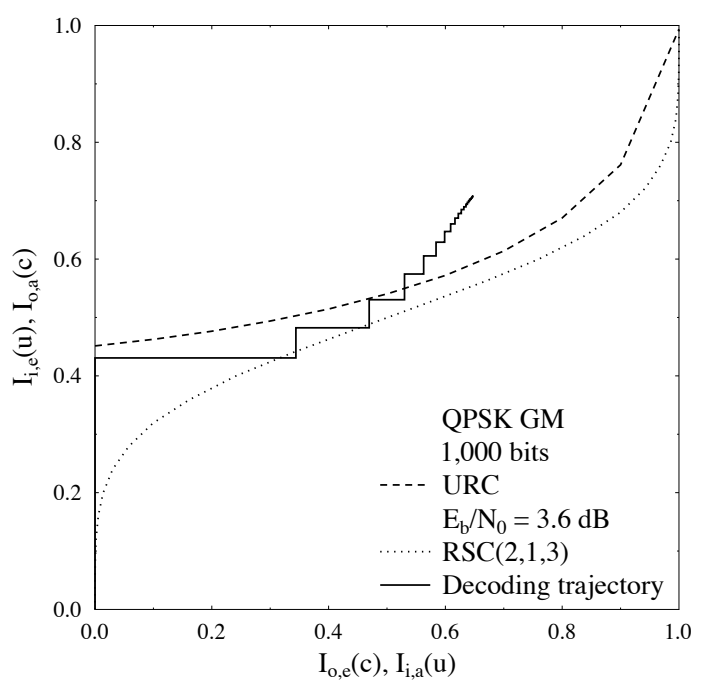

Fig. 31. Decoding trajectory of a $1 / 2$-rate, $K=3$ RSC-coded and URCprecoded scheme of Fig. 24 employing GM QPSK, while using an interleaver depth of 1,000 bits and operating at $E_{b} / N_{0}=3.6 \mathrm{~dB}$.

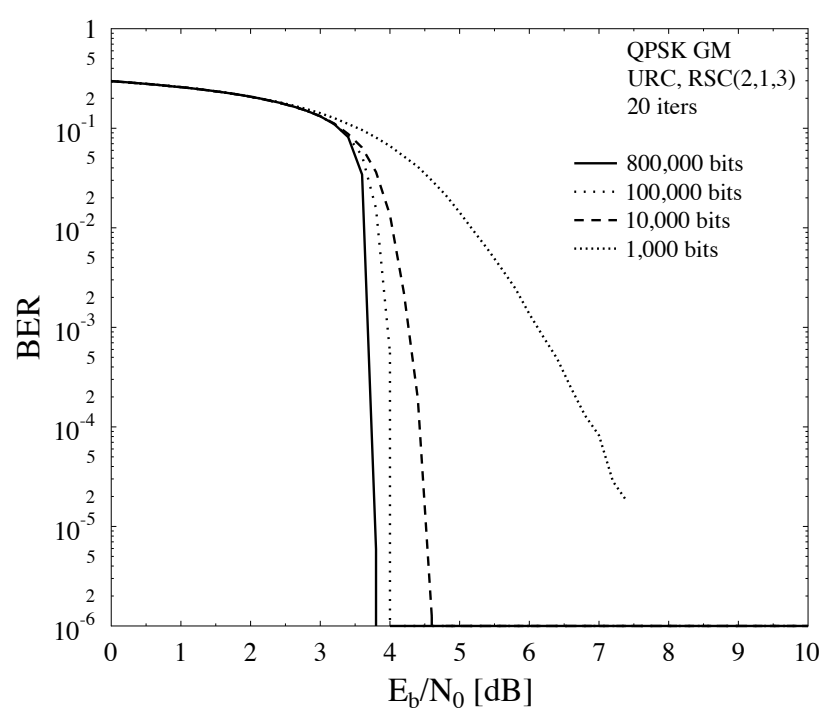

Fig. 32. BER Performance comparison of the 1/2-rate, $K=3$ RSC-coded and URC-precoded scheme of Fig. 24 employing GM QPSK, while varying the interleaver length.

a large number of iterations since the tunnel will become narrow. This suggests that near-capacity operation imposes a high complexity.

\section{Effect of interleaver depth on the accuracy of the EXIT chart prediction}

At the beginning of Section III, we mentioned the assumptions used for the application of the EXIT charts, namely that the apriori LLRs are fairly uncorrelated and the PDF of the apriori LLRs is Gaussian. These assumptions are valid, when using sufficiently high interleaver lengths. Below we will show the effect of varying the interleaver length on the accuracy of EXIT chart predictions.

Figs. 29, 30 and 31 record the Monte-Carlo simulation based decoding trajectory of the iteratively detected system 


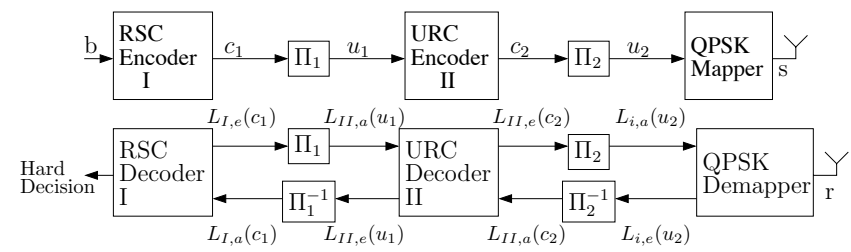

Fig. 33. Block diagram of a three-stage iteratively decoded system using QPSK modulation in conjunction with URC and RSC codes, which includes the additional interleaver $\Pi_{2}$ in comparison to Fig. 24.

of Fig. 24, while operating at $E_{b} / N_{0}=3.6 \mathrm{~dB}$ associated with interleaver depths of 100,000 bits, 10,000 bits and 1,000 bits, respectively. The decoding trajectory of Fig. 29 employs an interleaver depth of 100,000 bits, where the decoding trajectory is different from that observed in Fig. 28. In other words, the system employing a shorter interleaver requires more iterations to reach the highest point of intersection between the EXIT curves of the inner URC decoder and the outer RSC decoder. Additionally as $I_{i, a}(\mathbf{u})$ increases, the Monte-Carlo decoding trajectory starts to deviate from the EXIT curves of the URC or RSC decoders, because the LLR distribution is no longer Gaussian for high $I_{i, a}(\mathbf{u})$ values.

The Monte-Carlo simulation based decoding trajectories shown in Fig 30 and Fig. 31 became even more different from the EXIT chart predictions because shorter interleavers are used and thus the assumptions stipulated at the beginning of Section III become even less accurate. More explicitly, when using shorter interleavers, the LLRs $L_{i, a}(\mathbf{u})$ are no longer near-Gaussian distributed. This implies that the EXIT chart prediction of having an open tunnel at $E_{b} / N_{0}>3.0 \mathrm{~dB}$ and hence a low BER at this $E_{b} / N_{0}$ value is no longer valid for low interleaver lengths. This is verified by the BER performance curves shown in Fig. 32.

The BER performance of the iteratively detected RSCcoded and URC precoded scheme of Fig. 24 using 20 iterations and varying the interleaver depth is shown in Fig. 32. As seen in the figure, upon increasing the interleaver depth from 1,000 bits to 10,000 bits, the system's performance dramatically improves. Upon further increasing the interleaver depth from 10,000 bits to 100,000 bits, the attainable performance improves, but not as much as upon increasing it from 1,000 to 10,000 bits. Furthermore, increasing the interleaver depth beyond 100,000 bits fails to significantly improve the achievable system performance, as seen in Fig. 32, while comparing the BER curves recorded for the interleaver lengths of 800,000 bits and 100,000 bits.

\section{E. EXIT chart analysis of a three-stage serially concatenated system and 3D EXIT charts}

The turbo principle of [16] was extended to multiple serially concatenated codes in [19]. The appeal of concatenated coding is that low-complexity iterative detection replaces the potentially more complex optimum non-iterative decoder, such as that of [77]. The concept of EXIT chart analysis has been extended to three-stage concatenated systems in $[30,36,41$, 42].

The two-stage iteratively decoded system of Fig. 24 can be further improved by employing an interleaver at the output of

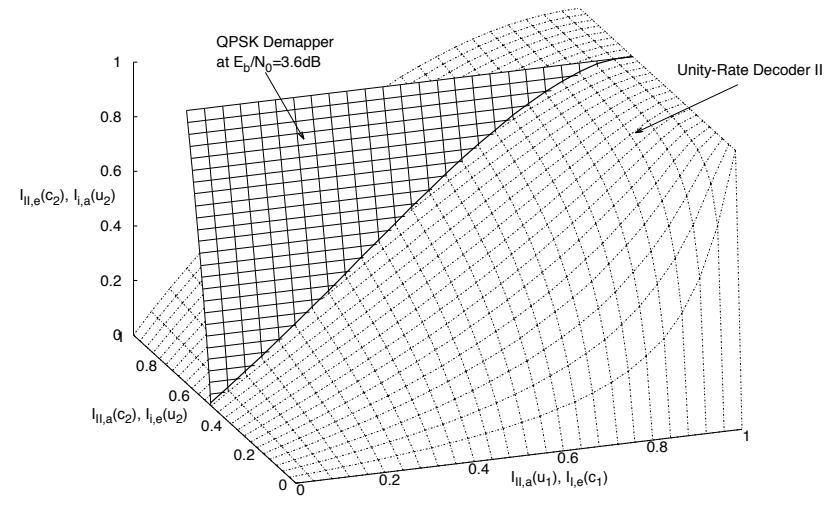

Fig. 34. 3D EXIT chart of Decoder II and the demapper of Fig. 33 at $E_{b} / N_{0}=3.6 \mathrm{~dB}$.

the URC, as shown in Fig. 33, in order to further randomising the bursty errors imposed by the channels characterised by both time and frequency selectivity. Furthermore, extrinsic soft information can be exchanged between the demapper and URC decoder resulting in a three-stage iteratively decoded system The schematic of the three-stage system is shown in Fig. 33, where the transmitted source bits $\mathbf{b}$ are encoded by the outer RSC Encoder I having a rate of $R_{I}$. Similarly to Fig. 24, the outer channel encoded bits $\mathbf{c}_{1}$ are then interleaved by the first random bit interleaver $\Pi_{1}$ of Fig. 33, where the randomly permuted bits $\mathbf{u}_{1}$ are fed through the URC Encoder II. The URC coded bits $\mathbf{c}_{2}$ at the output of the URC encoder are interleaved by the second random bit interleaver $\Pi_{2}$, as shown in Fig. 33, producing the permuted bits $\mathbf{u}_{2}$. After bit interleaving, the bits are mapped to QPSK symbols for transmission.

Similarly to Section III-B we consider transmission over a temporally correlated narrowband Rayleigh fading channel associated with a normalised Doppler frequency of $f_{D}=f_{d} T_{s}=0.01$. As shown in Fig. 33, the received complexvalued symbols $\mathbf{r}$ are decoded by the QPSK demapper. Then, iterative demapping/decoding is carried out by exchanging extrinsic information between the QPSK demapper, URC SISO Decoder II and RSC SISO Decoder I. The iterative process is performed for a number of consecutive iterations. During the last iteration, only the LLR values $L_{I, e}(\mathbf{b})$ of the original uncoded systematic information bits $\mathbf{b}$ are required, which are passed to a hard decision decoder in order to determine the estimated transmitted source bits, as shown in Fig. 33.

As seen from Fig. 33, the input of the URC Decoder II is constituted by the a priori input $L_{I I, a}\left(\mathbf{c}_{2}\right)$ and the a priori input $L_{I I, a}\left(\mathbf{u}_{\mathbf{1}}\right)$ provided by the demapper and Decoder I after bit deinterleaving, respectively. Therefore, the EXIT characteristic of Decoder II may be described by the following two EXIT functions [24,42]:

$$
\begin{aligned}
& \left.I_{I I, e}\left(\mathbf{c}_{\mathbf{2}}\right)=T_{I I, c_{2}}\left[I_{I I, a}\left(\mathbf{u}_{\mathbf{1}}\right), I_{I I, a}\left(\mathbf{c}_{\mathbf{2}}\right)\right)\right] \\
& I_{I I, e}\left(\mathbf{u}_{\mathbf{1}}\right)=T_{I I, u_{1}}\left[I_{I I, a}\left(\mathbf{u}_{\mathbf{1}}\right), I_{I I, a}\left(\mathbf{c}_{\mathbf{2}}\right)\right]
\end{aligned}
$$

which are illustrated by the $3 \mathrm{D}$ surfaces drawn in dotted lines 


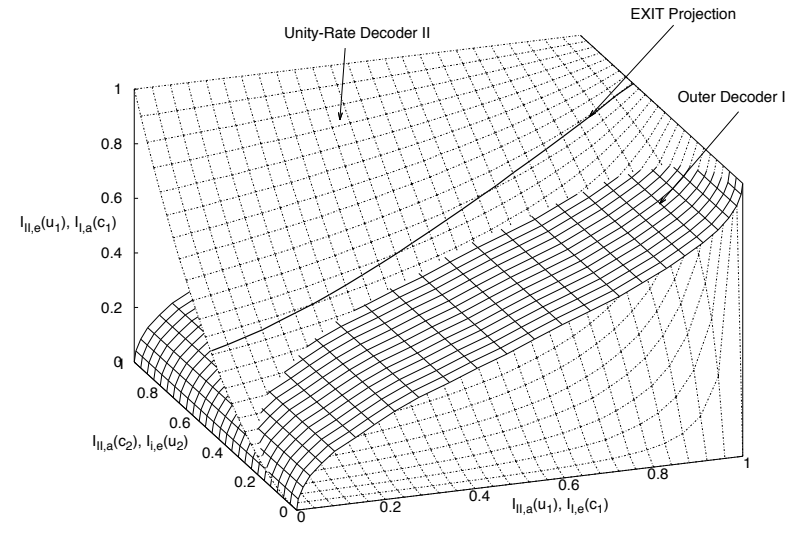

Fig. 35. 3D EXIT chart of Decoder II and Decoder I of Fig. 33, also showing the EXIT projection from Fig. 34.

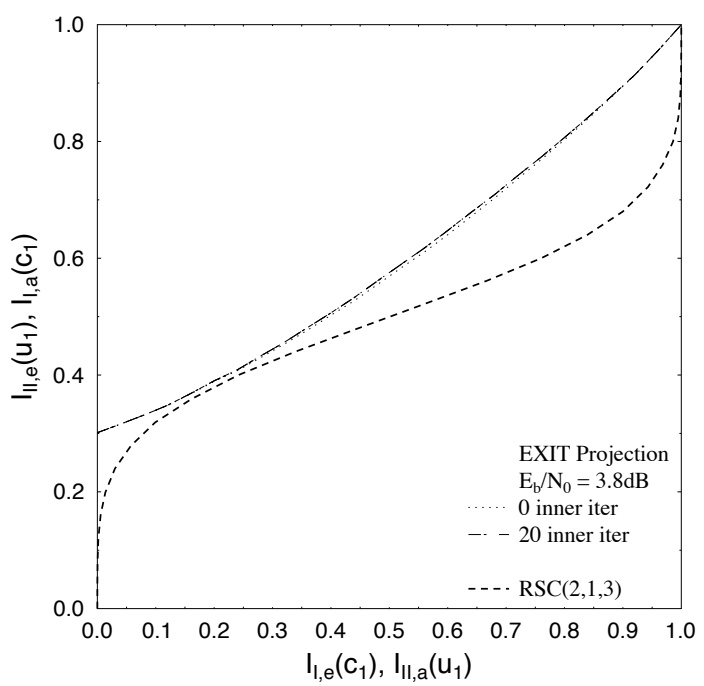

Fig. 36. 2D projection of the EXIT charts of Fig. 35 for the three-stage system of Fig. 33, when employing AGM aided QPSK, while using an interleaver depth of 800,000 bits.

in Fig. 34 and Fig. 35, respectively. On the other hand, the EXIT characteristic of the demapper as well as that of Decoder I are each dependent on a single a priori input, namely on $L_{i, a}\left(u_{2}\right)$ and $L_{I, a}\left(c_{1}\right)$, respectively, both of which are provided by the URC Decoder II after appropriately ordering the bits by the interleavers, as seen in Fig. 33. The EXIT characteristic of the demapper is also dependent on the $E_{b} / N_{0}$ value. Consequently, the corresponding EXIT functions drawn for the demapper and Decoder I, respectively, may be written as:

$$
\begin{aligned}
I_{I, e}\left(\mathbf{u}_{\mathbf{2}}\right) & =T_{i, u_{2}}\left[I_{i, a}\left(\mathbf{u}_{\mathbf{2}}\right), E_{b} / N_{0}\right] \\
I_{I, e}\left(\mathbf{c}_{\mathbf{1}}\right) & =T_{I, c_{1}}\left[I_{I, a}\left(\mathbf{c}_{\mathbf{1}}\right)\right]
\end{aligned}
$$

which are illustrated by the 3D surfaces drawn in solid lines in Fig. 34 and Fig. 35, respectively.

As observed in Fig. 34 and Fig. 35, it is somewhat cumbersome to interpret the 3D EXIT charts. Fortunately, their unique and unambiguous 2D representations can be deduced, which may be interpreted more readily. More explicitly, the intersection of the surfaces seen in Fig. 34 is shown as a thick

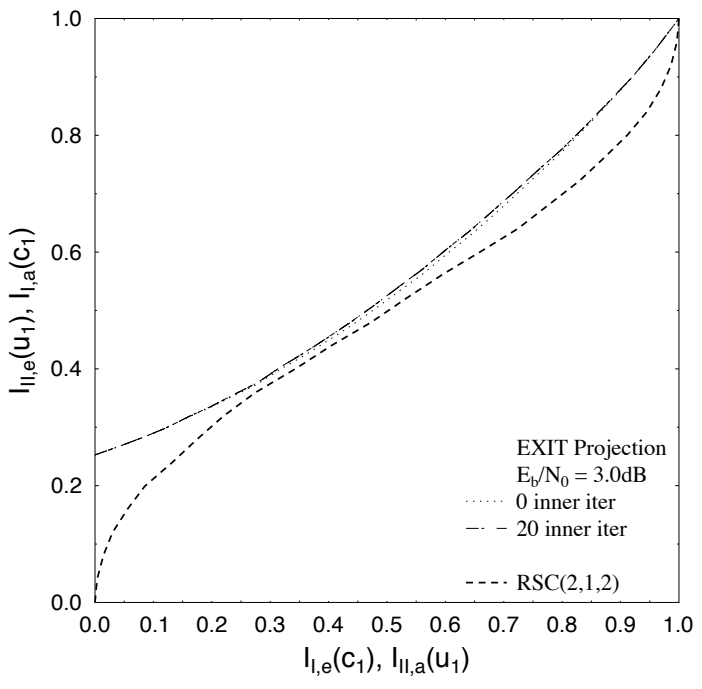

Fig. 37. 2D projection of the EXIT charts of the three-stage system of Fig. 33 when employing the AGM aided QPSK, while using an interleaver depth of 800,000 bits and an RSC with constraint length $K=2$ at $E_{b} / N_{0}=3.0 \mathrm{~dB}$.

solid line, which characterises the achievable performance when exchanging mutual information between the demapper and the URC Decoder II for different fixed values of $I_{I I, a}\left(\mathbf{u}_{1}\right)$ gleaned for Decoder I and spanning the range of $[0,1]$. Each $\left[I_{I I, a}\left(\mathbf{u}_{1}\right), I_{I I, a}\left(\mathbf{c}_{2}\right), I_{I I, e}\left(\mathbf{c}_{2}\right)\right]$ point belonging to the line of intersection in Fig. 34 uniquely specifies a point $\left[I_{I I, a}\left(\mathbf{u}_{1}\right), I_{I I, a}\left(\mathbf{c}_{2}\right), I_{I I, e}\left(\mathbf{u}_{1}\right)\right]$ in the 3D space of Fig. 35, according to the EXIT function of Equation (19). Therefore, the line corresponding to the $\left[I_{I I, a}\left(\mathbf{u}_{1}\right), I_{I I, a}\left(\mathbf{c}_{2}\right), I_{I I, e}\left(\mathbf{c}_{2}\right)\right]$ points along the thick line of Fig. 34 is projected to the solid line shown in Fig. 35. This projected EXIT curve may be written as

$$
I_{I I, e}\left(\mathbf{u}_{1}\right)=T_{I I, u_{1}}^{p}\left[I_{I I, a}\left(\mathbf{u}_{1}\right), E_{b} / N_{0}\right] .
$$

The intersection of the surfaces in Fig. 34, shown as a thick solid line, characterises the best achievable performance, when exchanging MI between the demapper and the URC Decoder II for different fixed values of $I_{I I, a}\left(\mathbf{u}_{1}\right)$ spanning the range of $[0,1]$. Therefore, the line corresponding to the $\left[I_{I I, a}\left(\mathbf{u}_{1}\right), I_{I I, a}\left(\mathbf{c}_{2}\right), I_{I I, e}\left(\mathbf{c}_{2}\right)\right]$ points along the thick line of Fig. 34 is projected to the solid line shown in Fig. 35, while the $2 \mathrm{D}$ projection of the solid line in Fig. 35 at $I_{I I, a}\left(\mathbf{c}_{2}\right)=0$ onto the plane spanned by the lines $\left[I_{I I, a}\left(\mathbf{u}_{1}\right), I_{I I, e}\left(\mathbf{u}_{1}\right)\right]$ and $\left[I_{I, e}\left(\mathbf{c}_{1}\right), I_{I, a}\left(\mathbf{c}_{1}\right)\right]$ is shown in Fig. 36 at $E_{b} / N_{0}=3.6 \mathrm{~dB}$. More explicitly, Fig. 36 records the 2D-projected EXIT curves for 0 and 20 inner iterations between the demapper and Decoder II. As observed from Fig. 36, when no inner iterations are employed, the system becomes essentially a two-stage arrangement as the one shown in Fig. 24 of Section III-C. According to Fig. 36, regardless whether 0 or 20 inner iterations are employed between the demapper and the URC decoder II, the projected curve has more or less the same shape and an open tunnel is attained for $E_{b} / N_{0}=3.6 \mathrm{~dB}$. This implies that a three-stage system relying on the configuration of Fig. 33 may be replace by a simpler two-stage system, which is capable of attaining the same performance.

On the other hand, the three-stage system of Fig. 33 
employing no inner iterations is equivalent to the two-stage system of Fig. 24. However, observe that the shape of the EXIT curve recorded for the three-stage system in Fig. 36 is different from that seen in Fig. 25, becuase the system of Fig. 36 is using AGM aided QPSK, while that of Fig. 25 is using GM aided QPSK.

Furthermore, according to the shape of the curve in Fig. 36, it is possible to use a different RSC code with a different constraint length or a different generator polynomial and improve the attainable system performance. This is demonstrated in Fig. 37, where a RSC code having a constraint length of $K=2$ is used. Observe in Fig. 37 that there is an open tunnel at $E_{b} / N_{0}=3.0 \mathrm{~dB}$, which means that it is possible to attain an infinitesimally low BER performance at $E_{b} / N_{0}=3.0 \mathrm{~dB}$. However, observe in Fig. 37 that the open tunnel is a narrow one and hence it requires numerous iterations for the decoding trajectory to reach the $(1.0,1.0)$ point of perfect convergence. This example demonstrated how powerful EXIT charts can be in optimising the achievable system performance without using exhaustive Monte-Carlo simulations.

\section{F. Extending the EXIT charts}

EXIT chart analysis was invoked for visualising the convergence behaviour of both two- and three-stage serially concatenated systems, in the previous sections. The analysis may be readily extended to parallel-concatenated systems as follows. When considering the decoder of the parallel concatenated system shown in Fig. 1, the extrinsic output LLRs $E\left(u_{1}\right)$ of Decoder 1 constitute the a priori input LLRs $A\left(u_{2}\right)$ for Decoder 2 after interleaving. Similarly, the extrinsic output LLRs $E\left(u_{2}\right)$ of Decoder 2 become the a priori input LLRs $A\left(u_{1}\right)$ of Decoder 1 after deinterleaving. Hence, the MI of the extrinsic output LLRs of Decoder 1 are the same as the MI of the a priori input LLRs of Decoder 2. The same is valid for the MI of the extrinsic LLRs of Decoder 2 and the a priori LLRs of Decoder 1. This implies that an EXIT chart can be generated for the parallel concatenated system of Fig. 1 by plotting the MI of $E\left(u_{1}\right)$ versus the MI of $A\left(u_{1}\right)$ for Decoder 1 and plotting the MI of $A\left(u_{2}\right)$ versus the MI of $E\left(u_{2}\right)$ for Decoder 2.

It is also possible to consider extending the serial concatenation of soft-input soft-output components beyond a threestage concatenated system. Before describing how a fourstage serially concatenated system can be analysed using EXIT charts, it is worth discussing whether there is any substantial performance gain justifying the complexity of an extra iterative stage. As shown in Fig. 36 and Fig. 37, the gain achieved by using a three stage system is less than $0.5 \mathrm{~dB}$ compared to the two-stage iteratively decoded system. However, this is attained at the expense of adding the complexity and delay of a new stage of iteration. Hence, using a multiple concatenated stages would not produce any significant gain, despite significantly increasing both the complexity and delay of the system. Furthermore, if we were to consider a fourstage serially concatenated system, then we would need four 3D EXIT charts in order to analyse the system, which makes it quite complicated for producing any useful analysis.

\section{SyStem CAPACITY AND THE MAXIMUM ACHIEVABLE} RATE

The capacity of a single-input-single-output AWGN channel was quantified by Shannon in 1948 [3,78]. Since then, substantial research efforts have been invested in finding channel codes that would produce an arbitrarily low probability of error. Shannon's channel capacity was only defined for Continuous-input Continuous-output Memoryless Channels (CCMC) [69], where the channel's input is constituted by a continuous-amplitude discrete-time Gaussian-distributed signal and the capacity is only restricted by either the signalling energy or the bandwidth [79]. By contrast, in the context of discrete-amplitude QAM [5] and PSK [69] signals, we encounter a Discrete-input Continuous-output Memoryless Channel (DCMC) [69].

It was argued in $[38,80]$ that the maximum achievable throughput of the system is equal to the area under the EXIT curve of the inner code, provided that the bit stream input to the demapper has independently and uniformly distributed bits, the channel is an erasure channel, the inner code has a unity rate and the MAP algorithm is used for decoding. Assuming that the area under the EXIT curve of the inner decoder, i.e. the demapper in the system of Fig. 11 and the URC decode in the system of Fig. 24, is represented by $\mathcal{A}_{i}$, then the maximum achievable rate for the outer code specified at a particular $E_{b} / N_{0}$ value is given by [38]:

$$
R_{\max }=\mathcal{A}_{i}\left(E_{b} / N_{0}\right) \text {. }
$$

In other words, if $\mathcal{A}_{i}$ is calculated for different $E_{b} / N_{0}$ values, the maximum achievable bandwidth efficiency $\eta_{\max }$ may be formulated as a function of the $E_{b} / N_{0}$ value as follows

$$
\begin{aligned}
\eta_{\max }\left(\underline{\left.E_{b} / N_{0}\right)}\right. & =B \cdot R_{\text {system }} \cdot R_{\max } \\
& \approx B \cdot R_{\text {system }} \cdot \mathcal{A}_{i}\left(E_{b} / N_{0}\right)[\mathrm{bit} / \mathrm{sec} / \mathrm{Hz}]
\end{aligned}
$$

where $B$ is the number of bits per modulated symbol and $R_{\text {system }}$ is the rate of transmission of the system. For example, in a single-antenna-aided system we have $R_{\text {system }}=1$, while $R_{\text {system }}=1 / 2$ for a four-antenna-aided space-time block coded system [81]. Additionally, $E_{b} / N_{0}$ and $E_{b} / N_{0}$ are related to each other as follows:

$$
\underline{E_{b} / N_{0}}=E_{b} / N_{0}+10 \log \left(\frac{R_{o}}{\mathcal{A}_{i}\left(E_{b} / N_{0}\right)}\right)[\mathrm{dB}],
$$

where $R_{o}$ is the original outer code rate used for generating the EXIT curve of the inner decoder/demapper corresponding to the different $\mathcal{A}_{i}$ values. A simple procedure may be used for calculating the maximum achievable bandwidth efficiency of Equation (25) for $E_{b} / N_{0} \in\left[\rho_{\min }, \rho_{\max }\right]$, where $\left[\rho_{\min }, \rho_{\max }\right]$ represents the range of $E_{b} / N_{0}$ values, where the EXIT chart was evaluated, assuming that $\epsilon$ is a small constant.

Algorithm 1 Maximum Achievable Bandwidth Efficiency Estimation using EXIT Charts:

Step 1: Set $R_{o}$ to the code rate of the channel encoder used when generating the EXIT charts.

Step 2: Set $E_{b} / N_{0}=\rho_{\min } \mathrm{dB}$.

step 3: $\quad$ Get $I_{i, e}(\mathbf{b})=T_{i}\left(I_{i, a}(\mathbf{b}), E_{b} / N_{0}\right)$ from the evaluated EXIT chart at $E_{b} / N_{0}$ value. 


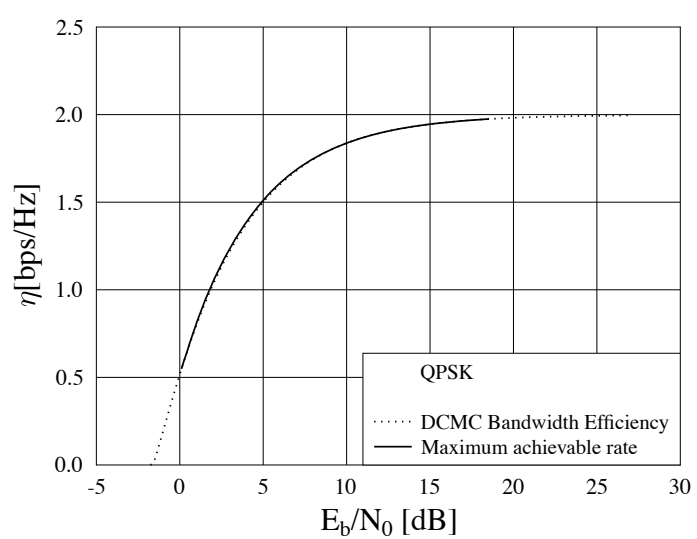

Fig. 38. Comparison of the DCMC bandwidth efficiency and the maximum achievable rate obtained using EXIT charts of a single-antenna-aided QPSK modulated system of Fig. 11.

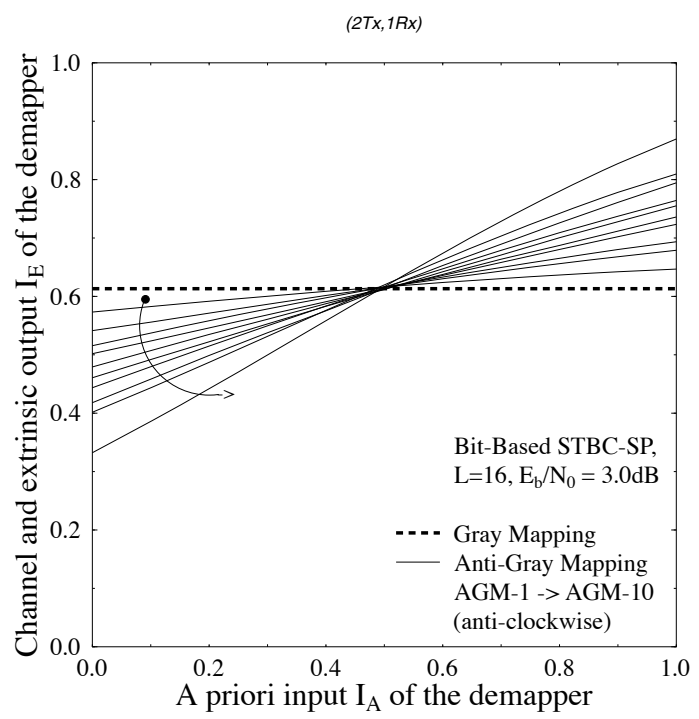

Fig. 39. Sphere packing demapper extrinsic information transfer characteristics for different bits to sphere-packing symbol mapping schemes at $E_{b} / N_{0}=3.0 \mathrm{~dB}$ for $L=16$ (c) Hanzo et. al. [1].

Step 4: $\quad$ Calculate $\quad \mathcal{A}_{i}\left(E_{b} / N_{0}\right)=$ $\int_{0}^{1} T_{i}\left(i, E_{b} / N_{0}\right) \mathrm{d} i$

Step 5: Calculate $E_{b} / N_{0}$ using Equation (25).

Step 6: Save $\eta_{\max } \overline{\left(E_{b} / N_{0}\right)}$ of Equation (25).

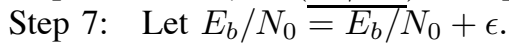
If $E_{b} / N_{0} \leq \rho_{\max } \mathrm{dB}$, go to Step 3 .

Step 8: Output $\eta_{\max }\left(\underline{E_{b} / N_{0}}\right)$ from Step 6.

Observe that $\rho_{\min }$ and $\rho_{\max }$ are adjusted accordingly, in order to produce the desired range of the resultant $E_{b} / N_{0}$ values. Furthermore, the output of Algorithm 1 is independent of the specific choice of $R_{o}$, since Equation (25) would always adjust the $E_{b} / N_{0}$ values, regardless of $R_{o}$.

The channel capacity curve of the single-antenna aided QPSK modulated system of Fig. 11 is shown in Fig. 38. The figures portrays both the DCMC bandwidth efficiency curve, evaluated according to [69], as well as the maximum achievable rate of the system derived from the EXIT curves according to Algorithm 1. Observe in Fig. 38 that the maximum achievable rate of the system derived from the EXIT curves is almost perfectly matching the DCMC

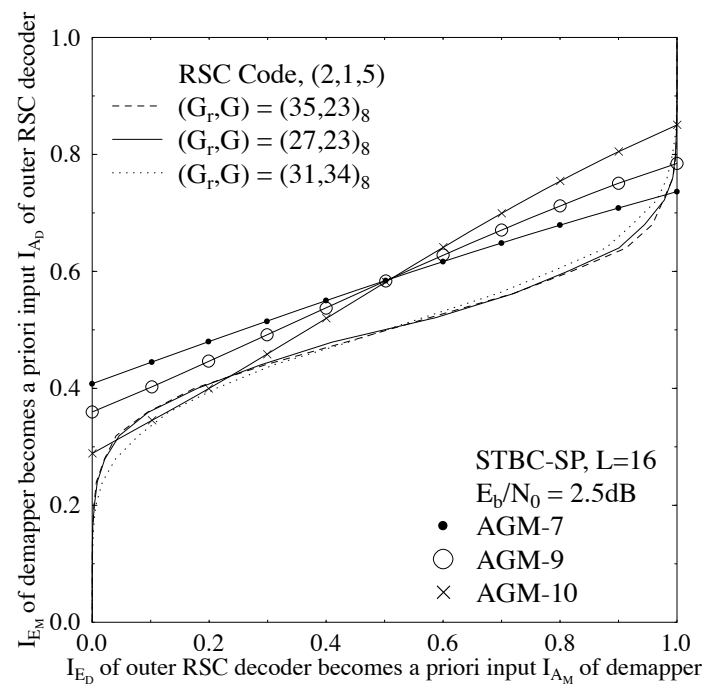

Fig. 40. EXIT chart of turbo-detected STBC-SP systems employing the AGM-7, AGM-9 and AGM-10 schemes of Fig. 39 in combination with outer RSC codes having a constraint length $K=5$ and different GPs (C) Hanzo et. al. [1].

bandwidth efficiency curve. Note that the maximum achievable rate obtained from the EXIT chart was proven mathematically for the family of binary erasure channels [80]. Nonetheless, similar experimentally verified trends have been observed for both AWGN and Inter-Symbol-Interference (ISI) contaminated channels $[34,38]$, provided that APP-based decoders are used for all decoder blocks [80].

\section{ApPliCATIONS OF EXIT CHARTS}

\section{A. Optimisation of sphere packing constellation mapping}

In [1] a multi-dimensional modulation scheme was introduced, which was referred to as Sphere Packing (SP) modulation combined with twin-antenna aided Space-Time Block Coding (STBC) $[81,82]$. SP modulation is capable of maximising the coding advantage of the transmission scheme by jointly designing and detecting the sphere-packed STBC system.

In [1], different GM and AGM schemes were proposed and optimised using EXIT charts. It was shown that EXIT charts can be used for selecting the best configuration of the constellation mapping in conjunction with diverse inner and outer code GPs and constraint lengths.

Fig. 39 shows the extrinsic information transfer characteristics of the SP demapper in conjunction with a SP signalling alphabet having $L=16$ symbols and different bit-to-symbol mapping schemes. As expected, GM does not provide any iteration gain upon increasing the MI at the input of the demapper. However, using a variety of different AGM schemes [57] results in different EXIT characteristics, as illustrated by the different slopes seen in Fig. 39. There are a total of 16factorial (16!) different mapping schemes. Having different legitimate bit-to-SP symbol mapping schemes provides us with the flexibility for selecting the most appropriate mapping for a specific system design with the aid of EXIT charts. This is shown in Fig. 40, where the EXIT charts of three possible SP AGM schemes are plotted in conjunction with three possible 


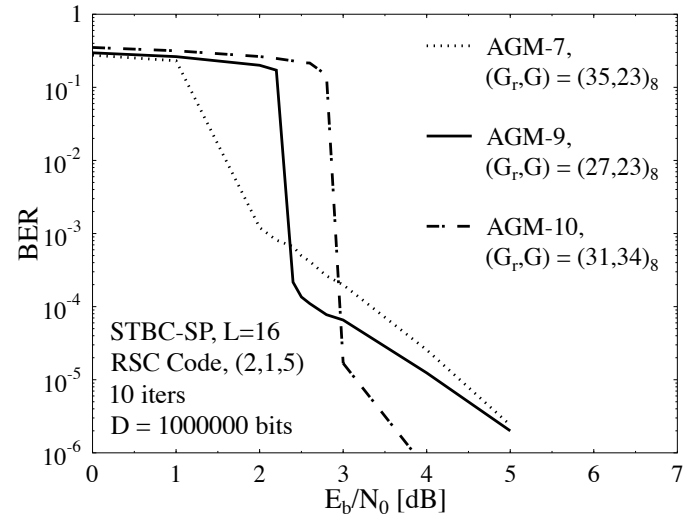

Fig. 41. BER performance of the AGM-7, AGM-9 and AGM-10 based RSC-coded STBC-SP schemes of Fig. 39 in conjunction with $L=16$ (C) Hanzo et. al. [1].

outer RSC code EXIT curves. As shown in Fig. 40, the AGM-10 scheme requires a higher SNR to generate an open tunnel than the AGM-9 modulated system, which in turn requires a higher SNR for attaining an open tunnel than the AGM-7 based system. This indicates that according to the EXIT chart prediction of Fig. 40, the AGM-7 based scheme exhibits a turbo cliff at a lower $E_{b} / N_{0}$ value than the others, when combined with the specific RSC code having the octal generator polynomials of $\left(G_{r}, G\right)=(35,23)_{8}$. However, the AGM-7 based scheme will acquire the highest BER floor, since the intersection point between the EXIT curves of the inner and outer codes is the lowest amonst the three AGM schemes considered. Therefore, different AGM schemes may be combined with specific RSC codes for the sake of designing systems satisfying specific criteria, such as for example attaining an early convergence or a lower BER floor. The BER performance comparison of the systems using the different AGM scheme used in Fig. 40 are shown in Fig. 41.

On the other hand, given the flexibility of the different AGMs, it is possible to optimise a three-stage iteratively detected system similar to that shown in Fig. 33 for SP modulation. More details about this issue can be found in [1].

EXIT charts and error floor: It is possible to relate the error floor observed in Fig. 41 to the corresponding EXIT curve in Fig. 40. As shown in Fig. 40, the EXIT curve of the demapper employing AGM-7 intersects with the EXIT curve of the outer decoder at a point lower than those for the demapper employing AGM-9 and AGM-10. This translates, as shown in Fig. 41, to a higher error floor for AGM-7 than AGM-9 and for AGM-10. This can be explained by referring to Fig. 18, where lower extrinsic LLRs result in higher BER and hence a higher error floor. The same can be said for comparing the error floors of the systems employing AGM-9 and AGM-10.

Fig. 18 shows clearly that a lower output extrinsic LLR of the outer decoder results in a lower BER. This means that the lower the intersection point between the inner and outer decoder EXIT curves, the higher the BER, hence resulting in a higher error floor. Furthermore, in order to get a lower error floor, the output extrinsic MI of the outer decoder, which corresponds to the intersection point between the EXIT curves

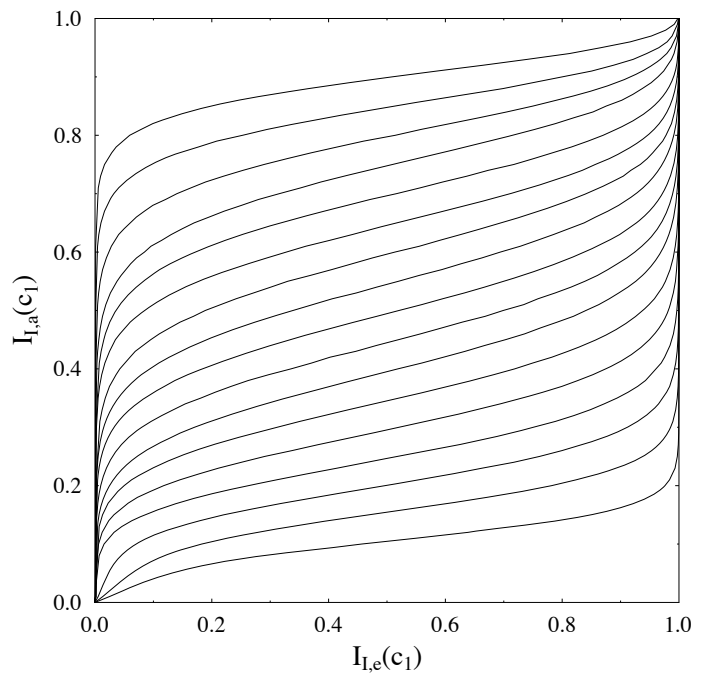

Fig. 42. EXIT functions of the seventeen $K=5$ subcodes in the IrCC, which have rates in the range $[0.1,0.9]$, which is confirmed by the area under their curves.

of the inner and outer decoders, should be as high as 0.998 as shown in Fig. 18.

\section{B. Design of irregular codes using EXIT charts}

As described in Section III, a narrow but marginally open EXIT-tunnel represents a near-capacity performance [38]. Therefore, IrCC were proposed in $[34,38]$ for the sake of appropriately shaping the EXIT curves by minimising the area within the EXIT tunnel using curve fitting of the EXIT charts.

For the sake of illustrating the IrCC concept further, we consider the three-stage system of Fig. 33 as an example. Let $\mathcal{A}_{I}$ and $\overline{\mathcal{A}}_{I}$ be the areas under the EXIT-curve of Decoder I and its inverse, respectively. Similarly, the area $\mathcal{A}_{p}$ is defined as that under the EXIT projection curve. It was observed in $[34,83]$ that for the APP-based outer Decoder I, the area $\overline{\mathcal{A}}_{I}$ may be approximated by $\overline{\mathcal{A}}_{I} \approx R_{I}$, where the equality $\overline{\mathcal{A}}_{I}=R_{I}$ was later shown in [80] for the family of Binary Erasure Channels (BECs). The area property of $\overline{\mathcal{A}}_{I} \approx R_{I}$ implies that the lowest SNR convergence threshold occurs, when we have $\mathcal{A}_{p}=R_{I}+\epsilon$, where $\epsilon$ is an infinitesimally small number.

Observe in Fig. 36 and Fig. 37 that there is a wide open tunnel between the EXIT curve of the outer decoder and the EXIT projection curve, especially for $I<0.2$ and $I>0.6$. This implies that the BER curve is farther from the achievable capacity than necessary. More quantitatively, the area under the EXIT projection curve is $\mathcal{A}_{p} \approx 0.57$ at $E_{b} / N_{0}=3.0 \mathrm{~dB}$, which is larger than the outer code rate of $R_{I}=0.50$. Therefore, according to Fig. 36 and Fig. 37 and to the area property of $\overline{\mathcal{A}}_{I} \approx R_{I}$, a lower $E_{b} / N_{0}$ convergence threshold may be attained. In other words, the EXIT curve of the outer code should match the EXIT projection curve more closely. Hence IrCCs can be invoked as an outer code that exhibit flexible EXIT characteristics, which can be optimised for more closely matching the EXIT projection curve of Fig. 36 and Fig. 37, converting the near-capacity code optimisation optimisation to a simple curve-fitting problem. 


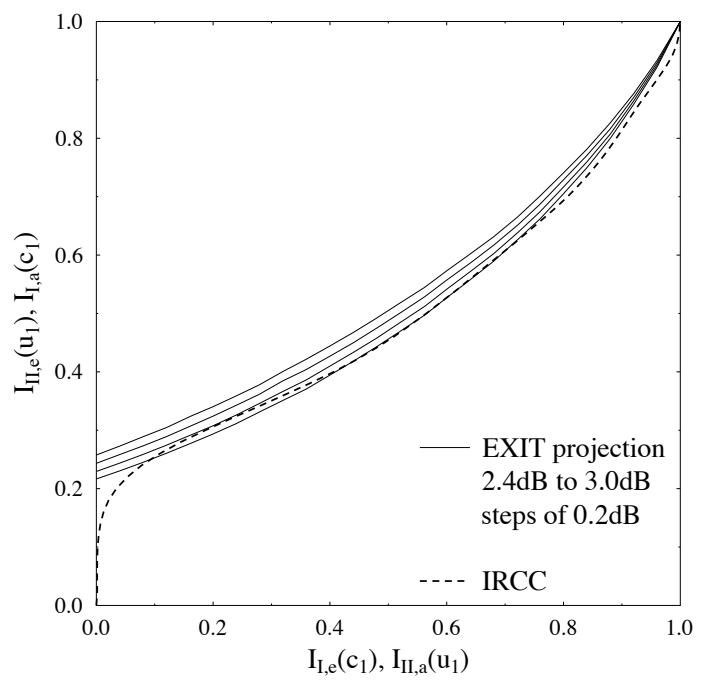

Fig. 43. EXIT chart of the two-stage system shown in Fig. 24 in conjunction with AGM based QPSK, where the outer RSC code was replaced by the IrCC code-components characterised in Fig. 42.

An IrCC scheme constituted by a set of 17 subcodes was constructed in [38] from a systematic 1/2-rate memory-4 mother code defined by the octally represented GPs $\left(G_{r}, G\right)=$ $(31,27)_{8}$. Each of the 17 subcodes have a different code rate $R_{I}^{i}, \forall i \in[1,17]$, where puncturing was employed to obtain the rates of $R_{I}^{i}>0.5$ and the code rates of $R_{I}^{i}<0.5$ were created by adding more GPs and by puncturing. The two additional GPs employed in [38] are defined by the octally represented GPs of $G_{1}=(35)_{8}$ and $G_{2}=(35)_{8}$, where the resultant 17 subcodes have coding rates spanning the range of $[0.1,0.9]$. Each of the 17 subcodes encodes a specific fraction of the uncoded bits determined by the weighting coefficient $\alpha_{i}, i=1, \ldots, P$. Assuming an overall average code rate of $R_{I}=0.5$, the following conditions must be satisfied:

$$
\sum_{i=1}^{17} \alpha_{i}=1, \quad R_{I}=\sum_{i=1}^{17} \alpha_{i} R_{I}^{i}, \quad \text { and } \quad \alpha_{i} \in[0,1], \quad \forall i .
$$

The EXIT function corresponding to the IrCC may be constructed from the EXIT functions of the 17 subcodes. More specifically, the EXIT function of the IrCC is the weighted superposition of the 17 EXIT functions, as follows [38]

$$
T_{I, c_{1}}\left[I_{I, a}\left(c_{1}\right)\right]=\sum_{i=1}^{17} \alpha_{i} T_{I, c_{1}}^{i}\left[I_{I, a}\left(c_{1}\right)\right] .
$$

Again, Fig. 42 shows the EXIT functions of the 17 subcodes used in [38]. Hence the coefficients $\alpha_{i}$ are optimised with the aid of the iterative algorithm of [34], so that the EXIT curve of the resultant IrCC closely matches the EXIT projection curve at that specific $E_{b} / N_{0}$ value, where we have $\mathcal{A}_{p} \approx 0.50$. It is observed that we have $\mathcal{A}_{p} \approx 0.501$ at $E_{b} / N_{0}=2.4 \mathrm{~dB}$. However, observe in Fig. 43 that we have an open tunnel at $E_{b} / N_{0}=2.6 \mathrm{~dB}$, indicating that this $E_{b} / N_{0}$ value is close to the lowest attainable convergence threshold, when employing a 1/2-rate outer code. Fig. 43 also shows the EXIT curve of the resultant IrCC. It may be inferred from Fig. 43 that an open convergence tunnel is formed around $E_{b} / N_{0}=2.6 \mathrm{~dB}$.
This implies that according to the predictions of the EXIT chart of Fig. 43, the iterative decoding process is expected to converge at $E_{b} / N_{0}=2.6 \mathrm{~dB}$. However, observe in Fig. 43 that the open tunnel at $E_{b} / N_{0}=2.6 \mathrm{~dB}$ is quite narrow and thus it requires a large number of iterations to converge at $E_{b} / N_{0}=2.6 \mathrm{~dB}$.

\section{Multi-dimensional constellation labelling}

It is widely recognised that the choice of a specific bit-tosymbol mapping scheme or a beneficial constellation labelling is an influential factor, when designing Bit-Interleaved Coded Modulation using Iterative Decoding (BICM-ID) for the sake of achieving a high iteration gain [24, 84, 85]. Hence, in [84] an EXIT chart based flexibility measure was introduced for analysing the effect of expanding the constellation to a higherdimensional space by jointly transmitting a pair of symbols of a classic two-dimensional constellation.

In order to attain a near-capacity performance, the SNR value corresponding to the turbo cliff has to be as low as possible. The crucial point at the receiver is that the concatenated detectors/demappers/decoders are SISO decoders that are capable of accepting and delivering probabilities or soft values, where the extrinsic information of the soft-output of one detector/demapper/decoder is passed on to the other detector/demapper/decoder to be used as its a priori input.

Several papers studied the multi-dimensional constellation labelling demonstrating that it was beneficial to invoke multidimensional modulation in the context of both TCM and BICM [76, 86-88]. However, these papers did not quantify the achievable improvement, neither did they detail whether the improvement was merely due to the increased number of bits used by the combined symbols allowing for more flexibility in the design of the constellation, or due to the beneficial multi-dimensional labelling. In [84], a flexibility measure was proposed based on EXIT charts, where investigations were carried out into the effect of expanding the constellation to more dimensions as well as to quantify the benefits of increasing the classic constellation order. It was shown in [84] that the flexibility improvement of the bit-to-symbol mapping offered by expanding the modulation constellation to a higherdimensional space, when communicating over a Rayleigh channel was mainly due to the increased number of bits-perjoint-symbol. Nonetheless, useful EXIT flexibility improvements may also be obtained by expanding the modulation constellation to a higher-dimensional space, especially when communicating over an independent fading based Rayleigh channel [84].

\section{Symbol-based EXIT charts}

The analysis in Section III was presented in the context of iterative decoding at the bit level. However, it was shown in [32] that a reduced transmit power may be required, when symbol-based rather than bit-based iterative decoding is employed.

The convergence behaviour of bit-based iterative decoding was studied in Section III. EXIT charts have been extended to iterative non-binary coding schemes in [33], where a histogram-based approximation of the extrinsic information's 
PDF was required in order to compute the MI. Moreover, the evaluation of the extrinsic information's histogram becomes computationally demanding for a large number of bits per symbol. Hence a reduced-complexity method of computing non-binary EXIT charts was proposed in [43], which dispenses with the above-mentioned histogram computation and may be considered to constitute a generalisation of the approach presented in [44].

Motivated by the performance improvements reported in $[32,45,89,90]$, a symbol-based iterative scheme was proposed in [1], where it was shown that the non-binary turbodetection aided scheme is capable of providing further performance improvements over the equivalent bit-based turbodetected scheme.

\section{E. Coded Modulation}

As mentioned in Section I of this paper, one of the objectives for the design of digital communications is the efficient exploitation of the available bandwidth in order to accommodate the ever-increasing traffic demand. Hence coded modulation was proposed, where it is possible to insert parity bits into the bit sequence and absorb them by expanding the signal constellation in order to accommodate the parity bits without expanding the bandwidth of the un-coded signal [4]. It has been shown in $[4,91]$ that the design of coded modulation schemes is affected by different design criteria. While a high squared free Euclidean distance is desired for Additive White Gaussian Noise (AWGN) channels, a high effective code length and minimum product distance are desired for fading channels [92]. Trellis-Coded Modulation (TCM) [91,93] was originally proposed for Gaussian channels, but later it was further developed for applications in mobile communications over fading channels $[92,94]$. Again, the philosophy of TCM schemes is that they combine channel coding and modulation in a way that the modulated signal constellation is extended to an increased number of constellation points, so that more bits per symbol can be transmitted for the sake of absorbing the parity bits, hence requiring no increase in the bandwidth [95]. Turbo Trellis-Coded Modulation (TTCM) [96] is another joint coding and modulation scheme that has a structure similar to that of the family of binary turbo codes [16], but it employs TCM schemes as component codes.

The coded modulation design and optimisation has been presented for AWGN channels [91,93], where the design criteria was that of acquiring a higher free Euclidean distance. Then TCM was redesigned for fading channels, where the criteria is that of attaining a minimum product distance [92]. Then, after the introduction of EXIT charts, they were used as a powerful tool in designing and optimising coded modulation techniques, especially because the bit-to-symbol mapping makes the TCM encoder structure more complicated than that of classic turbo codes [97]. In [33], the EXIT chart concept has been extended to the non-binary case, where serially concatenated systems having an inner TCM component and a space-time convolutional code were analysed. Furthermore, it was shown in [33] that the requirement of having a Gaussian distribution for the a priori LLRs of a decoder can only be really argued based on the properties of the extrinsic information, rather than those of the actual transmission channel. Afterwards, in [97] the approach of [33] was extended to TTCM relying on employing symbol-based EXIT charts. On the other hand, the non-binary EXIT chart technique proposed in [43] was shown to be well-suited to the analysis of non-binary iterative decoding schemes associated with a large index alphabet, such as for example a TTCM system employing a 32-QAM signal set. In [98], the EXIT chart was utilised in order to develop a design criterion for choosing the constituent codes of TTCM, where the optimization method was developed for constructing high-rate and high-performance serially concatenated TCM schemes.

The EXIT charts of Self-Concatenated Trellis Coded Modulation (SCTCM) [99] were presented in [100], where it was found that the symbol-based EXIT charts are fairly accurate in predicting the decoding convergence threshold, despite the inaccurate simplifying assumption that the extrinsic information and the systematic information of each encoded symbol are independent of each other. For example, it was shown in [100] that the EXIT chart analysis of SCTCM schemes allows the designer to determine the number of self-iterations required by the SCTCM decoder for achieving convergence and hence to attain a very low BER [95].

Another coded modulation scheme distinguishing itself by utilising bit-based interleaving in conjunction with Gray signal constellation labelling is referred to as Bit-Interleaved Coded Modulation (BICM) [101]. More explicitly, BICM combines conventional convolutional codes with several independent bit interleavers in order to increase the achievable diversity order of a code for transmission over fading channels [101]. The number of parallel bit interleavers equals the number of coded bits in a symbol for the BICM scheme proposed in [101]. The performance of BICM is better than that of TCM for transmission over uncorrelated or perfectly interleaved narrowband Rayleigh fading channels, but worse than that of TCM in Gaussian channels owing to the reduced Euclidean distance of BICM. Additionally, iterative joint decoding and demodulation assisted BICM (BICM-ID) was proposed in an effort to further increase the achievable system performance $[21,91,102-104]$, where the approach of BICM-ID is to increase the Euclidean distance of BICM and hence to exploit the full advantage of bit interleaving with the aid of soft-decision iterative decoding [22].

The convergence behaviour of BICM-ID was analysed in [105] for the case of imperfect channel state information, while communicating over fading channels. It was shown using EXIT charts that the correlation coefficient between the fading and its estimate is the key parameter affecting the decoding convergence. Additionally, it was shown that the decoding convergence can be controlled by striking a trade-off between the quality of channel estimation and the code rate. Furthermore, EXIT charts were also used for analysing BICMID systems over the an AWGN channel in [106], where the authors develop a generalised model for the a priori inputs. It was shown in [106] that the demapper and decoder transfer functions are sensitive to over-estimation of the noise variance. Additionally, in [107] a method was proposed for predicting the BERs of BICM-ID for all types of channels, which is based on EXIT charts and avoids extensive BER simulations. 
In [108], the iterative convergence of TCM, TTCM, BICM and DICM-ID was analysed using EXIT charts and a 3dimensional EXIT chart was proposed for studying the iterative convergence behaviour of the multi-stage decoding used in Multi-Level Coding (MLC) [109]. Furthermore, in [110] a near-capacity irregular BICM-ID (Ir-BICM-ID) scheme was designed, which adopted a design philosophy similar to that of the IrCC scheme of $[34,38]$. It was shown in [110] that the irregular design of the Ir-BICM-ID scheme pervades the three basic components of BICM-ID, namely the encoder, the unity-rate precoder and the bit-to-symbol mapper, where the proposed ir-BICM-ID schemes are capable of approaching the capacity of coded modulation. Furthermore, since the appropriate choice of the bit-to-symbol mapping, which is also often referred to as constellation labelling, is essential in the design of BICM-ID, EXIT charts were used to design this labelling for BICM-ID in [111].

\section{F. Combining EXIT chart and union bounds for system design and analysis}

According to the previous analysis, it has been shown how the EXIT charts can be used as a powerful technique of analysing and designing iteratively decoded system characterised by near-capacity performance. EXIT charts have been successfully applied in both bit-based as well as in symbol-based iteratively detected systems. In [112] the lowcomplexity symbol-based EXIT charts proposed in [43] and the corresponding BER union bound of Turbo Trellis Coded Modulation (TTCM) schemes [96] were employed in order to design new, near-capacity symbol-interleaved TTCM schemes. More specifically, new GPs were sought for the Trellis Coded Modulation (TCM) [93] component codes, based on both their decoding convergence and on the error floor of the TTCM decoder, rather than using the classic 'punctured' minimal distance criterion of the TCM component codes defined in [96]. In [112] the prime design criterion was that of finding a constituent TCM code, where the corresponding EXIT charts exhibit an open tunnel at the lowest possible SNR value, as well as having an acceptable error floor, where the latter was estimated by the truncated symbol-based union bound.

\section{G. EXIT band charts}

It has been shown in Section III that the EXIT chart and its prediction is accurate for long interleavers, where the LLR's PDF approaches the Gaussian distribution. This implies that the EXIT chart constitutes an accurate technique of predicting the convergence behaviour in the turbo-cliff region of a BER curve, when using long interleavers. In $[39,40]$, the EXIT band chart tool was proposed for analysing systems having shorter interleavers.

The EXIT charts are obtained by evaluating the average MI of the a priori $i$ and the extrinsic LLRs, while running several simulations with the aid of long interleavers. In this case, the variance of the different simulations is small. However, when running simulations for shorter interleavers, the MI transfer curve becomes different for each single simulation run even at the same $E_{b} / N_{0}$ value for both the inner and outer decoders. In this case, a band of EXIT curves is evaluated for both the inner as well as the outer decoders for each given $E_{b} / N_{0}$ value. The band of MI transfer curves is then referred to as the EXIT band [39], where the EXIT chart can be inferred by getting the average of the band. It was shown in [39] that as the interleaver length increases, the width of the EXIT band reduces until we arrive at the EXIT chart valid for long interleavers.

\section{H. Code design}

Since the introduction of turbo codes and iterative decoding [16], substantial research efforts have been invested in improving their performance as well as in reducing their complexity. For example, by choosing the best constituent encoder, a turbo code would achieve an improved performance without increasing its complexity. In [113], a parameter referred to as the effective free distance was introduced for optimising the design of parallel concatenated codes and it was shown that good constituent codes are those that have the maximum possible effective free distance [62]. The effective free distance can be defined as the lowest-weight codeword having the lowest for any weight-2 message [48]. In [113-115] a list of the best low-complexity constituent codes were presented based on maximising the effective free distance.

On the other hand, it was shown in [25] that the design of the constituent codes of turbo codes relying on iterative decoding based on the effective free distance substantially affects the code's performance in the error-floor region, whilst having only a modest effect on the convergence properties of the code and on its performance in the turbo cliff region. These statements may be readily confirmed with the aid of EXIT charts. Additionally, it was shown in [116] that there is a difference between the codes designed according to the noniterative Maximum Likelihood (ML) design criterion and those designed according to the iterative decoding criterion. For ML decoding, the design criterion is to maximise the effective free distance, while the design criterion for iterative decoding using EXIT charts is to find the lowest turbo cliff SNR. Hence, it was concluded in [116] that a trade-off has to be struck between both design criteria for the sake of designing good codes. For example, in [117] non-systematic turbo codes were designed, where the effective free distance was maximised in order to provide a better error floor performance and the EXIT charts were utilised for improving the turbo-cliff performance of the proposed non-systematic turbo codes.

\section{Further EXIT Chart Applications}

EXIT charts constitute a powerful semi-analytical tool that may be used for analysing any system, where there is an exchange of information. In a practical system relying on realistic channel estimation and synchronisation, the errors due to channel estimation and synchronisation imperfections may also be considered as an additional source of noise, hence the EXIT chart analysis may provide us with an indication of the degradation due to realistic imperfect channel estimation and synchronisation, without the need to embark on the timeconsuming Monte-Carlo simulations [118].

On the other hand, in a multi-user system supporting $N$ users $[119,120]$, where Multi-User Detection (MUD) is 
employed at the receiver, the EXIT charts have to be $(N+1)$ dimensional to analyse such a system, which makes its employment infeasible. In this case, we may assume however that all transmitters have the same transmission power. Then, an average EXIT chart can be adopted for the entire system in order to arrive at an indication of the average system performance, while varying the number of users, for example. Furthermore, it may be argued that EXIT charts can be used for estimating the complexity of an iteratively decoded system by predicting the number of iterations required for attaining the target BER performance.

Additionally, based on the EXIT-curve matching technique of IrCCs, Irregular Variable Length Coding (IrVLC) was proposed in [121] for near-capacity joint source and channel coding. In IrVLC a number of component Variable Length Coding (VLC) codebooks having different coding rates encode specific fractions of the input source symbol stream. EXIT charts are used for appropriately selecting these fractions to shape the IrVLC EXIT curve in order to match that of the inner decoder, which allows for near-capacity performance. In [121] an IrVLC was amalgamated with TCM, where iterative decoding was employed at the receiver for exchanging extrinsic information between the IrVLC and TCM decoders. On the other hand, an IrVLC was used in [122] combined with a Time Hopping (TH) Pulse Position Modulation (PPM)aided Ultra Wide-Band (UWB) Spread Spectrum (SS) impulse radio system. In [122] the TH PPM-aided UWB SS system was amalgamated with a URC in conjunction with the lossless IrVLC [121] joint source and channel coding in order to attain near-capacity performance. Additionally, in [123] iterative decoding of the IrVLC scheme concatenated with precoded Fast Frequency Hopping (FFH) M-ary Frequency Shift Keying (MFSK) was considered. It was shown in [123] that using the proposed scheme, an infinitesimally low BER may be achieved at low SNR values.

On the other hand, the EXIT chart matching technique was invoked for designing serially concatenated inner and outer codes in [124]. In [124] the inner code used was a URC, while the outer code was a VLC, where the irregular design was constituted by a variety of the inner and outer component codes and EXIT chart matching was used for minimising the area of the open EXIT tunnel between the inner and outer codes' EXIT curves. Hence, this facilitates operation at $E_{b} / N_{0}$ values that are closer to the channel's capacity bound. Additionally, another irregular design based on EXIT charts was proposed in [110], where a near-capacity Irregular BICM-ID (Ir-BICM-ID) scheme was proposed. The irregular design of the scheme uses the three components of BICM-ID, namely the encoder, the URC and the bit-to-symbol mapper. The proposed system was capable of approaching the capacity of coded modulation.

Furthermore, a capacity-approaching cooperative spacetime coding scheme employing irregular design for a twinrelay aided network was proposed in [125], where a successive relaying protocol was employed for the sake of recovering the multiplexing loss imposed by a half-duplex three-terminal network. Additionally, near-capacity coding was employed by the proposed system, where the cooperative space-time codes used at the source and the relays were jointly designed with the aid of EXIT charts for attaining a near-relayingchannel-capacity performance. As a further advance, in [126] an Irregular Distributed Space-Time (Ir-DST) coding scheme designed for near-capacity cooperative communications was presented. The proposed system's effective throughput was maximised with the aid of a joint source-and-relay mode design procedure. In the system presented in [126], an IrCC was serially concatenated with a URC and a STBC at the source node for the sake of approaching the corresponding source-to-relay link capacity. EXIT charts were used for optimising the IrCC. On the other hand, another IrCC was serially concatenated with an identical STBC at the relay node. The relay's IrCC was re-optimised using EXIT charts for the sake of approaching the relay channel's capacity and also to maximise the relay's coding rate, hence maximising the effective throughput. At the destination node, a threestage iterative decoding scheme was constructed for achieving decoding convergence to an infinitesimally low BER.

Since the introduction of turbo-code-aided Hybrid Automatic Repeat reQuest (HARQ) schemes, research focused on their complexity reduction. In [127], an early stopping strategy was proposed for a turbo HARQ scheme, which results in significant complexity reduction, while maintaining a high throughput. Further improvements were also proposed in [127], where a new Deferred Iterations (DI) strategy was designed to take into account the prevalent channel conditions. More explicitly, the proposed DI strategy delays the commencement of the turbo-decoding until an open EXIT chart tunnel appears, leading to a successful decoding operation.

\section{DESIGN GUIDELINES}

In the age of smart phones and tablet PCs, the main driving force behind the advances in wireless communications is the promise of seamless global mobility and flawless high-rate wireless multimedia services. This is required, while meeting a range of contradicting design challenges such as those included in the illustration of Fig. 44. For example, it is possible to both increase the effective throughput and simultaneously to reduce the BER, if an increased implementation complexity and a higher power consumption may be tolerated to facilitate more sophisticated signal processing [2].

In the following we will focus on the design of channel coding utilising the EXIT chart analysis tool, while reflecting on the design trade-offs of Fig. 44. Considering a given coding rate, we focus on the following design trade-offs: BER, coding gain, interleaving delay and complexity in terms of the number of decoding iterations. Fig. 45 shows the achievable BER of the RSC-coded and URC-precoded scheme of Fig. 24 for a variable number of decoding iterations and a variable interleaver length at $E_{b} / N_{0}$ of $3.6 \mathrm{~dB}$. As shown in Fig. 45, one more iteration is necessitated by the system employing an interleaver length of 100,000 bits in order to achieve the same BER as that of the system employing an interleaver of length 800,000 bits. Furthermore, the systems using interleaver lengths of 1,000 and 10,000 bits acquire little to no gain upon increasing the number of iterations at $E_{b} / N_{0}$ of $3.6 \mathrm{~dB}$. Relating this to the design factors of Fig. 44, it is shown that increasing the complexity in terms of the number of decoding iterations as well as increasing 


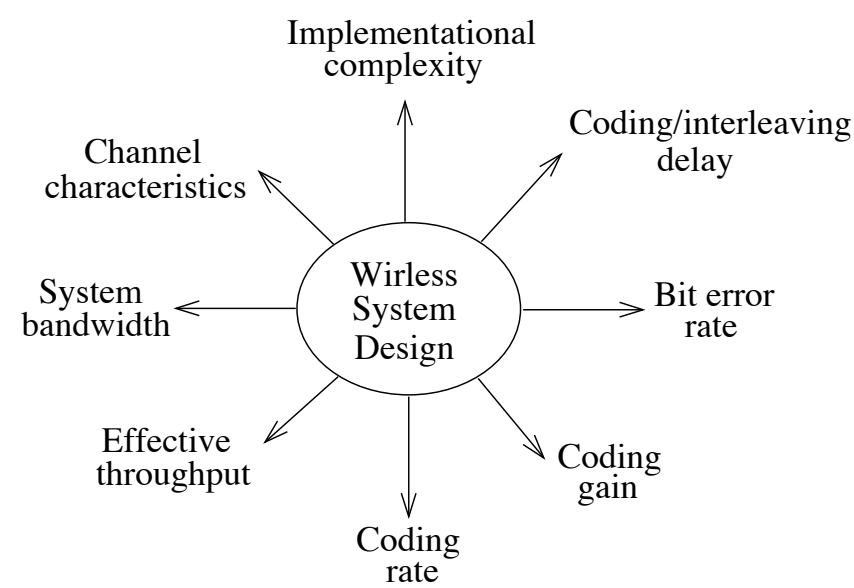

Fig. 44. Factors affecting the design of channel coding and modulation schemes (c)Hanzo et. al. [4].

the interleaver delay, it is possible to attain an improved BER performance.

The results shown in Fig. 45 can be related to the EXIT charts and decoding trajectories of Figs. 28, 29, 30 and 31. It has been shown in Section III-D that when sufficiently long interleavers are used, the decoding trajectory can reach the $(1.0,1.0)$ point of perfect decoding convergence to a vanishingly low BER.

Furthermore, it has been mentioned in Section III-B2 that two different methods can be used for evaluating the MI. One of the methods computes the MI by evaluating the histogram based approximation of $p_{E}[25,26,46]$ and then applying Equation (13) or alternatively by using the time averaging method [35] of Equations (15) and (17). The histogram-based and the averaging methods stipulate different assumptions, as described in Section III-A, although they both assume that the ' 0 's and ' 1 's of the encoder input bit sequence are equiprobable. When the LLRs satisfy the consistency condition, then the averaging method gives more accurate results as compared to the histogram-based method, which on the other hand works better when using long interleavers. The consistency condition is normally satisfied when all the component in the system are optimal and are using no approximation such as the MAP or Log-MAG algorithms. This does not mean that the two methods will result in different EXIT curves. The two methods can be used to verify that the design is sound by making sure that the EXIT curves using the two methods are matching.

It was shown in [128] that when employing non-optimal decoders relying on several approximations, the EXIT curve of the inner URC decoder does not reach the $(1.0,1.0)$ point, although the URC uses a recursive encoder. It was also shown that even if the LLRs of the decoders are clipped at a maximum value, in order to eliminate any chance for them to saturate, this does not result in the correct EXIT curve for the LLRs satisfying the consistency condition. It was concluded in [128] that this phenomenon was due to the assumption used in the demapper and decoders namely that the data was Gaussian distributed, even though this assumption was inaccurate. Therefore, a LLR pre-processing technique was proposed in order to have LLRs output by the demapper

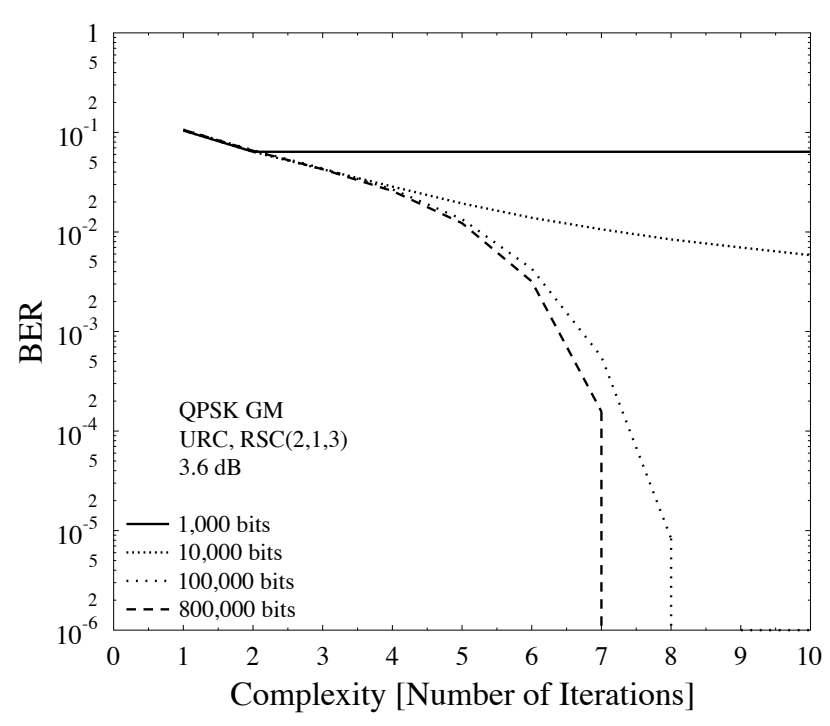

Fig. 45. Achievable BER of the RSC-coded and URC-precoded scheme of Fig. 24 in combination with the outer $\operatorname{RSC}(2,1,3)$ and operating for at $E_{b} / N_{0}$ of $3.6 \mathrm{~dB}$ with different interleaver lengths and number of iterations

satisfying the consistency condition.

\section{CONCLUSIONS}

In order for any communications system to attain a low BER at SNRs close to the channel capacity, it is essential to invoke channel coding and iterative decoding. Iterative decoding can be done for parallel as well as serially concatenated systems. Substantial efforts have been invested in optimising the performance of concatenated codes in order to improve the slopes of their BER curves and to attain a near-capacity performance. Recently, significant efforts have also been dedicated to studying the convergence behaviour of iterative decoding. EXIT charts were proposed as a powerful semi-analytical tools devised for analysing the convergence behaviour of iteratively decoded systems, where MI between the data bits at the transmitter and the soft values at the receiver were used for describing the exchange of extrinsic information between the constituent decoders.

The main objective of employing EXIT charts is to predict the convergence behaviour of iterative decoding by analysing the evolution of the MI exchange between the constituent decoders in consecutive iterations. In this paper, we have shown how the EXIT charts can be used for analysing and designing iteratively decoded systems using two-stage and three-stage iteratively decoded serially concatenated systems as design examples. The EXIT chart properties can be summarised as follows:

- Ideally, in order for the exchange of extrinsic information between the inner and the outer decoders to converge for the sake of achieving a vanishingly low BER at a specific $E_{b} / N_{0}$ value, the EXIT curve of the inner decoder recorded at the $E_{b} / N_{0}$ value of interest and that of the outer RSC decoder should not intersect before reaching the $(1.0,1.0)$ point.

- This implies that given $I_{a}=1.0$, we have $I_{e}=1.0$ and provided that this condition is satisfied, an open 
convergence tunnel appears in the EXIT chart. The narrower the tunnel, the more iterations are required for reaching the $(1.0,1.0)$ point and the closer the BER curve approaches the vertical line representing the channel capacity.

- If however, the two extrinsic MI transfer characteristics intersect at a point close to the vertical line at $I_{i, a}=1.0$ - rather than at the $(1.0,1.0)$ point - then a low BER may still be achieved, although it will remain higher than the BER of the schemes having an intersection at the $(1.0,1.0)$ point. These types of EXIT tunnels are referred to here as semi-convergent tunnels.

- The EXIT chart based decoding convergence predictions are usually verified by the Monte-Carlo simulation based iterative decoding trajectory as detailed in Section III-B4.

- In order for the EXIT charts of the inner and outer decoders to intersect at the $(1.0,1.0)$ point, a recursive inner decoder component is needed for maximising the interleaver gain and for avoiding the formation of a BER floor. This can be achieved by employing a URC, which has an IIR and hence it efficiently randomises the extrinsic information without increasing the interleaver delay and without reducing the throughput.

- The application of EXIT charts is based on two assumptions, namely on having a high interleaver length, which ensures that the a priori LLR values are fairly uncorrelated and the probability density function of the a priori LLR values is Gaussian. It has been shown in Section III-D that when using short interleavers, these two assumptions become less accurate. This implies that the EXIT chart prediction of having an open tunnel and hence a low BER at the specific $E_{b} / N_{0}$ is no longer valid.

- EXIT charts can also be used for predicting the convergence behaviour of three-stage iteratively decoded systems. In this case, 3D EXIT charts are generated, where 2D EXIT charts can be generated by projecting the intersection between the 3D EXIT curves of the "inner and intermediate" or the "intermediate and outer" decoder onto a 2D EXIT chart with the outer or inner decoder EXIT charts, respectively.

- The maximum achievable throughput of a system is equal to the area under the EXIT curve of the inner decoder as detailed in Section IV.

\section{LIST OF ACRONYMS}

\begin{tabular}{ll} 
AGM & Anti-Gray Mapping \\
AWGN & Additive White Gaussian Noise \\
BCJR & Bahl-Cocke-Jelinek-Raviv algorithm \\
BEC & Binary Erasure Channel \\
BER & Bit Error Rate \\
BICM & Bit-Interleaved Coded Modulation \\
BICM-ID & BICM relying on Iterative Detection \\
CC & Convolutional Codes \\
CCMC & Continuous-input Continuous-output Memo- \\
\multicolumn{2}{c}{ ryless } \\
DCMannels \\
DI less Channel \\
Discrete-input Continuous-output Memory-
\end{tabular}

EXIT Extrinsic Information Transfer

FFH Fast Frequency-Hopping

GM Gray Mapping

GP Generator Polynomials

HARQ Hybrid Automatic Repeat reQuest

IIR Infinite Impulse Response

Ir-BICM-ID Irregular BICM-ID

IrCC Irregular Convolutional Codes

Ir-DST Irregular Distributed Space-Time coding

IrVLC Irregular Variable Length Coding

ISI Inter-Symbol-Interference

LLR Logarithmic-Likelihood Ratio

Log-MAP Log Maximum Aposteriori Probability

MAP Maximum A Posteriori

MFSK M-ary Frequency-Shift Keying

MI Mutual Information

MLSE

MUD

PDF

PLR

PPM

RSC

SISO

SNR

SP

SS

STBC

TCM

$\mathrm{TH}$

TTCM

URC

UWB

Maximum Likelihood Sequence Estimation

Multi-User Detection

Probability Density Function

Packet Loss Ratio

Pulse Position Modulation

Recursive Systematic Convolutional

Soft-Input Soft-Output

Signal to Noise Ratio

Sphere Packing

Spread Spectrum

Space-Time Block Coding

Trellis Coded Modulation

Time Hopping

Turbo Trellis Coded Modulation

Unity-Rate Code

Ultra Wide-Band

VLC Variable Length Coding

\section{REFERENCES}

[1] L. Hanzo, O. Alamri, M. El-Hajjar and N. Wu, Near-Capacity MultiFunctional MIMO Systems: Sphere-Packing, Iterative Detection and Cooperation. John Wiley \& Sons - IEEE Press, 2009.

[2] L. Hanzo, M. El-Hajjar and O. Alamri, "Near-Capacity Wireless Transceivers and Cooperative Communications in the MIMO Era: Evolution of Standards, Waveform Design, and Future Perspectives," Proc. IEEE, vol. 99, pp. 1343-1385, August 2011.

[3] C. E. Shannon, "A mathematical theory of communication," Bell Systems Technical Journal, vol. 27, pp. 623-656, October 1948.

[4] L. Hanzo, T.H. Liew, B.L. Yeap, R.Y.S. Tee, S.X. Ng, Turbo Coding, Turbo Equalisation and Space-Time Coding: EXIT-Chart-Aided NearCapacity Designs for Wireless Channels, Second Edition. John Wiley \& Sons - IEEE Press, 2011.

[5] L. Hanzo, S.X. Ng, T. Keller and W. Webb, Quadrature amplitude modulation: From basics to adaptive trellis-coded, turbo equalised and space-time coded OFDM, CDMA and MC-CDMA systems, 2nd Edition. Chichester, England: John Wiley and Sons Ltd and IEEE Press, 2004.

[6] R.W. Hamming, "Error detecting and error correcting codes," Bell Systems Tech. Journal, vol. 29, pp. 41-56, 1950.

[7] P. Elias, "Coding for noisy channels," IRE Conv. Rept., pp. 37-47, 1955.

[8] J. Wozencraft, "Sequential decoding for reliable communications," IRE Nat. Conv. Rec., vol. 5, pp. 11-25, 1957.

[9] J. Wozencraft and B. Reiffen, Sequential Decoding. MIT Press, 1961.

[10] R. Fano, "A heuristic discussion of probabilistic decoding," IEEE Trans. Inf. Theory, vol. 9, pp. 64-74, April 1963.

[11] J. Massey, Threshold Decoding. MIT Press, 1963. 
[12] A. Viterbi, "Error bounds for convolutional codes and an asymptotically optimum decoding algorithm," IEEE Trans. Inf. Theory, vol. 13 pp. 260-269, April 1967.

[13] G. Forney, “The Viterbi algorithm,” Proc. IEEE, vol. 61, pp. 268-278, march 1973

[14] L. Bahl, J. Cocke, F. Jelinek and J. Raviv, "Optimal decoding of linear codes for minimizing symbol error rate," IEEE Trans. Inf. Theory, vol. 20 , no. 2 , pp. 284-287, 1974.

[15] G. Forney, Concatenated codes. Cambridge: MIT Press, 1966.

[16] C. Berrou, A. Glavieux and P. Thitimajshima, "Near Shannon limit error-correcting coding and decoding: Turbo-codes. 1," in IEEE International Conference on Communications, vol. 2, (Geneva), pp. 1064 1070, May 1993.

[17] C. Berrou, A. Glavieux, "Near optimum error correcting coding and decoding: turbo-codes," IEEE Trans. Commun., vol. 44, pp. 12611271, October 1996.

[18] D. Divsalar and F. Pollara, "Multiple turbo codes for deep-space communications," Telecommunications and Data Acquisition Progress Report 42-121, Jet Propulsion Laboratory, Pasadena, CA, May 1995.

[19] S. Benedetto and G. Montorsi, "Serial concatenation of block and convolutional codes," Electronics Letters, vol. 32, pp. 887-888, May 1996.

[20] G. Caire, G. Taricco and E. Biglieri, "Bit-interleaved coded modulation," IEEE Trans. Information Theory, vol. 44, pp. 927-946, May 1998.

[21] X. Li and J. A. Ritcey, "Bit-interleaved coded modulation with iterative decoding," IEEE Commun. Lett., vol. 1, pp. 169-171, November 1997.

[22] X. Li and J. A. Ritcey, "Bit-interleaved coded modulation with iterative decoding using soft feedback," IEE Electronics Letters, vol. 34 pp. 942-943, May 1998.

[23] M. Peleg, I. Sason, S. Shamai and A. Elia, "On interleaved differentially encoded convolutional codes," IEEE Trans. Inf. Theory, vol. 45, pp. 2572-2582, November 1999.

[24] S. ten Brink, "Designing iterative decoding schemes with the extrinsic information transfer chart," AE $\ddot{U}$ International Journal of Electronics and Communications, vol. 54, pp. 389-398, November 2000.

[25] S. ten Brink, "Convergence behavior of iteratively decoded parallel concatenated codes," IEEE Trans. Commun., vol. 49, no. 10, pp. 17271737, 2001.

[26] S. Ten Brink, "Code characteristic matching for iterative decoding of serially concatenated codes," Annals of Telecommunications, vol. 56, pp. 394-408, 2001.

[27] T. J. Richardson and R. Urbanke, "The capacity of low-density parity-check codes under message-passing decoding," IEEE Trans. Inf. Theory, vol. 47, pp. 599-618, February 2001.

[28] T.J. Richardson, A. Shokrollahi and R. Urbanke, "Design of capacityapproaching low-density parity-check codes," IEEE Trans. Inf. Theory, vol. 47, pp. 619-637, February 2001.

[29] S. Y. Chung, G. D. Forney, T. J. Richardson and R. Urbanke, "On the design of low-density parity-check codes within $0.0045 \mathrm{~dB}$ of the Shannon limit," IEEE Commun. Lett., vol. 5, pp. 58-60, February 2001.

[30] S. ten Brink, "Convergence of multi-dimensional iterative decoding schemes," in 35th Asilomar Conference on Signals, Systems, and Computers, vol. 1, (Pacific Grove, CA), pp. 270-274, November 2001.

[31] H. El Gamal and A.R. Hammons, "Analyzing the turbo decoder using the Gaussian approximation," IEEE J. Sel. Areas Commun., vol. 47, pp. 671-686, February 2001.

[32] B. Scanavino, G. Montorsi and S. Benedetto, "Convergence properties of iterative decoders working at bit and symbol level," in Proceed ings of IEEE Global Telecommunications Conference (GLOBECOM), vol. 2, (San Antonio, TX), pp. 1037-1041, November 2001.

[33] A. Grant, "Convergence of non-binary iterative decoding," in Proc. IEEE Global Telecommunications Conference (GLOBECOM), vol. 2, (San Antonio, USA), pp. 1058-1062, November 2001.

[34] M. Tüchler and J. Hagenauer, "EXIT charts of irregular codes," in Conference on Information Science and Systems, (Princtone, NJ), pp. 748-753, March 2002.

[35] M. Tüchler, S. ten Brink and J. Hagenauer, "Measures for tracing convergence of iterative decoding algorithms," in Proc. 4th International ITG Conference on Source and Channel Coding, (Berlin, Germany), pp. 53-60, 2002.

[36] M. Tüchler, "Convergence prediction for iterative decoding of threefold concatenated systems," in IEEE Global Telecommunications Conference (GLOBECOM ), vol. 2, pp. 1358-1362, November 2002.

[37] M. Tüchler, "Design of serially concatenated systems for long or short block lengths," in Proc. IEEE International Conference on Communications (ICC), vol. 4, (Anchorage Alaska, USA), pp. 29482952, May 2003.
[38] M. Tüchler, "Design of serially concatenated systems depending on the block length," IEEE Trans. Commun., vol. 52, pp. 209-218, February 2004

[39] J.W. Lee and R.E. Blahut, "Lower bound on BER of finite-length turbo codes based on EXIT characteristics," IEEE Commun. Lett., vol. 8, pp. 238-240, April 2004.

[40] J.W. Lee and R.E. Blahut, "Convergence Analysis and BER Performance of Finite-Length Turbo Codes," IEEE Trans. Commun., vol. 55, pp. 1033-1043, May 2007.

[41] F. Brännström, L. K. Rasmussen and A. Grant, "Optimal scheduling for multiple serially concatenated codes," in 3rd International Symposium on Turbo Codes and Related Topics, (Brest, France), pp. 383-386, September 2003.

[42] F. Brännström, L.K. Rasmussen and A.J. Grant, "Convergence analysis and optimal scheduling for multiple concatenated codes," IEEE Trans. Inf. Theory, vol. 51, no. 9, pp. 3354-3364, 2005.

[43] J. Kliewer, S.X. Ng and L. Hanzo, "Efficient Computation of EXIT Functions for Nonbinary Iterative Decoding," IEEE Trans. Commun., vol. 54, pp. 2133-2136, December 2006.

[44] I. Land, P. Hoeher and S. Gligorevic, "Computation of symbol-wise mutual information in transmission systems with $\log$ APP decoders and application to EXIT charts," in Proc. International ITG Conference on Source and Channel Coding (SCC), (Erlangen, Germany), pp. 195202, January 2004.

[45] D. Divsalar, S. Dolinar and F. Pollara, "Low complexity turbo-like codes," in 2nd International Symposium on Turbo Codes and Related Topics, (Brest, France), pp. 73-80, September 2000.

[46] S. Ten Brink, "Design of serially concatenated codes based on iterative decoding convergence," in Proc. 2nd International Symposium on Turbo Codes and Related Topics , (Brest, France), pp. 319-322, September 2000.

[47] S. ten Brink, "Convergence of iterative decoding," Electronics Letters, vol. 35, no. 13, pp. 1117-1119, 1999.

[48] S.J. Johnson, Iterative Error Correction: Turbo, Low-Density ParityCheck and Repeat-Accumulate Codes. Cambridge University Press, November 2009.

[49] J. Hagenauer, "The EXIT chart - introduction to extrinsic information transfer in iterative processing," in European Signal Processing Conference, (Vienna, Austria), pp. 1541-1548, September 2004.

[50] J.M. Walsh, P.A. Regalia and C.R. Johnson, "Turbo Decoding as Iterative Constrained Maximum-Likelihood Sequence Detection," IEEE Trans. Inf. Theory, vol. 52, pp. 5426-5437, December 2006.

[51] J. Hagenauer, "The turbo principle: Tutorial introduction and state of the art," in Proc. 1st International Symposium on Turbo Codes and Related Topics, (Brest, France), pp. 1-12, 1997.

[52] S. Benedetto, D. Divsalar, G. Montorsi and F. Pollara, "Serial concatenation of interleaved codes: performance analysis, design and iterative decoding," IEEE Trans. Inf. Theory, vol. 44, pp. 909-926, May 1998.

[53] S. Benedetto, D. Divsalar, G. Montorsi and F. Pollara, "Analysis, design and iterative decoding of double serially concatenated codes with interleavers," IEEE J. Sel. Areas Commun., vol. 16, pp. 231-244, February 1998.

[54] L. Hanzo, R.G. Maunder, J. Wang and L.-L. Yang, Near-Capacity Variable-Length Coding: Regular and EXIT Chart-Aided Irregular Designs. John Wiley \& Sons - IEEE Press, 2011.

[55] S. Huettinger and J. Hubber, "Design of multiple turbo codes with transfer characteristics of component codes," in Proc. Conference on Information Science and Systems, March 2002.

[56] C. Douillard, M. Jezequel, C. Berrou, A. Picart, P. Didier and A. Glavieux, "Iterative correction of intersymbol interference: turbo equalization," European Trans. Telecommunications, vol. 6, pp. 507-511, September/October 1995.

[57] S. ten Brink, J. Speidel and R.-H. Yan, "Iterative demapping and decoding for multilevel modulation," in IEEE Global Telecommunications Conference (GLOBCOM), vol. 1, pp. 579-584, 1998.

[58] M. Moher, "An iterative multiuser decoder for near-capacity communications," IEEE Trans. Commun., vol. 46, pp. 870-880, July 1998.

[59] R. Bauer and J. Hagenauer, "On variable length codes for iterative source/channel decoding," in Proc. Data Compression Conference (DCC), (Snowbird, UT), pp. 273-282, March 2001.

[60] M. Adrat, P. Vary and J. Spittka, "Iterative source-channel decoder using extrinsic information from softbit-source decoding," in IEEE International Conference on Acoustics, Speech and Signal Processing, pp. 2653-2656, May 2001.

[61] B. Vucetic, Y. Li, L.C. Perez and F. Jiang, "Recent Advances in Turbo Code Design and Theory," Proc. IEEE, vol. 95, pp. 1323-1344, June 2007. 
[62] S. Benedetto and G. Montorsi, "Unveiling turbo codes: some results on parallel concatenated coding schemes," IEEE Trans. Inf. Theory, vol. 42, pp. 409-428, March 1996.

[63] L.C. Perez, J. Seghers and D.J. Costello, Jr., "A distance spectrum interpretation of turbo codes," IEEE Trans. Inf. Theory, vol. 42, pp. 1698-1709, November 1996.

[64] R.G. Maunder and L. Hanzo, "Evolutionary Algorithm Aided Interleaver Design for Serially Concatenated Codes," IEEE Trans. Commun., vol. 59, pp. 1753-1758, July 2011.

[65] S. Dolinar and D. Divsalar, "Weight distributions for turbo codes using random and nonrandom permutations," tech. rep., JPL, 1995.

[66] F. Daneshgaran, M. Laddomada and M. Mondin, "Interleaver design for serially concatenated convolutional codes: theory and application," IEEE Trans. Inf. Theory, vol. 50, pp. 1177-1188, June 2004.

[67] J. Yu, M. Boucheret, R. Vallet, A. Duverdier and C. Mesnager, "Interleaver design for serial concatenated convolutional codes," IEEE Commun. Lett., vol. 8, pp. 523-525, August 2004.

[68] P. Robertson, "Illuminating the structure of code and decoder of parallel concatenated recursive systematic (turbo) codes," in IEEE Global Telecommunications Conference (GLOBECOM), vol. 3, pp. 12981303, November 1994.

[69] J. Proakis and M. Salehi, Digital Communications. McGraw-Hill, 2008.

[70] T.M. Cover and J.A. Thomas, Elements of information theory. New York: Wiley, 1991.

[71] J. Hagenauer, E. Offer and L. Papke, "Iterative decoding of binary block and convolutional codes," IEEE Trans. Inf. Theory, vol. 42, no. 2, pp. 429-445, 1996.

[72] V.B. Balakirsky, "Joint source-channel coding with variable length codes," in IEEE International Symposium on Information Theory, (Ulm, Germany), p. 419 , June 1997.

[73] P. Robertson, E. Villebrun and P. Hoeher, "A comparison of optimal and sub-optimal MAP decoding algorithms operating in the Log domain," in Proc. International Conference on Communications, (Seattle, USA), pp. 1009-1013, June 1995.

[74] L. Hanzo, J.P. Woodard and P. Robertson, "Turbo Decoding and Detection for Wireless Applications," Proc. IEEE, vol. 95, pp. 1178 1200, June 2007.

[75] N.H. Tran and H.H. Nguyen,, "Improving the performance of QPSK BICM-ID by mapping on the hypercube," in IEEE 60th Vehicular Technology Conference VTC-Fall, vol. 2, pp. 1299-1303, September 2004.

[76] N.H. Tran and H.H. Nguyen, "Design and performance of BICMID systems with hypercube constellations," IEEE Trans. Wireless Commun., vol. 5, pp. 1169-1179, May 2006.

[77] M. Breiling and L. Hanzo, "The super-trellis structure of turbo codes," IEEE Trans. Inf. Theory, vol. 46, pp. 2212-2228, September 2000.

[78] C. E. Shannon, "A mathematical theory of communication," Bell Systems Technical Journal, vol. 27, pp. 379-423, July 1948.

[79] S.X. Ng and L. Hanzo, "On the MIMO channel capacity of multidimensional signal sets," IEEE Trans. Veh.r Technol., vol. 55, no. 2, pp. 528-536, 2006.

[80] A. Ashikhmin, G. Kramer and S. ten Brink, "Extrinsic information transfer functions: model and erasure channel properties," IEEE Trans. Inf. Theory, vol. 50, no. 11, pp. 2657-2673, 2004.

[81] H. Jafarkhani, Space-time coding: Theory and practice. Cambridge University Press, 2005.

[82] S.M. Alamouti, "A simple transmit diversity technique for wireless communications," IEEE J. Sel. Areas Commun., vol. 16, no. 8, pp. 1451-1458, 1998.

[83] A. Ashikhmin, G. Kramer and S. ten Brink, "Extrinsic information transfer functions: A model and two properties," in 36th Annual Conference on Information Sciences and Systems (CISS), (Princeton, NJ, USA), March 2002.

[84] O. Alamri, B. Poupart, M. El-Hajjar, S.X. Ng and L. Hanzo, "On multidimensional BICM-ID constellation labelling," in IEEE International Conference on Communications (ICC), pp. 1-5, May 2010.

[85] A. Chindapol and J. A. Ritcey, "Design, analysis, and performance evaluation for BICM-ID with square QAM constellations in Rayleigh fading channels," IEEE J. Sel. Areas Commun., vol. 19, pp. 944-957, May 2001.

[86] S. Bäro, "Turbo-detection in MIMO systems: bit labeling and precoding," in 5th International ITG Conference on Source and Channel Coding, (Erlangen, Germany), January 2004.

[87] F. Simoens, H. Wymeersch, H. Bruneel and M. Moeneclaey, "Multidimensional mapping for bit-interleaved coded modulation with BPSK/QPSK signaling," IEEE Commun. Lett., vol. 9, pp. 453-455, May 2005.
[88] A. S. Mohammed, W. Hidayat and M. Bossert, "Multidimensional 16-QAM constellation labeling of BI-STCM-ID with the Alamouti scheme," in Proc. IEEE Wireless Communications and Networking Conference (WCNC), (Las Vegas, USA), April 2006.

[89] W. Su, Z. Safar and K. J. Liu, "Space-time signal design for timecorrelated Rayleigh fading channels," in Proc. IEEE International Conference on Communications (ICC), vol. 5, (Anchorage, Alaska), pp. 3175-3179, May 2003.

[90] M. C. Davey and D. J. MacKay, "Low density parity check codes over GF(q)," IEEE Commun. Lett., vol. 2, pp. 165-167, June 1998.

[91] S.X. Ng, T.H. Liew, L.-L. Yang and L. Hanzo, "Comparative study of TCM, TTCM, BICM and BICM-ID schemes," in IEEE Veh. Technol. Conference, vol. 4, pp. 2450-2454, 2001.

[92] D. Divsalar and M.K. Simon, "The design of trellis coded MPSK for fading channels: performance criteria," IEEE Trans. Commun., vol. 36 , pp. 1004-1012, September 1988.

[93] G. Ungerboeck, "Channel coding with multilevel/phase signals," IEEE Trans. Inf. Theory, vol. 28, pp. 55-67, January 1982.

[94] D. Divsalar and M.K. Simon, "The design of trellis coded MPSK for fading channels: set partitioning for optimum code design," IEEE Trans. Commun., vol. 36, pp. 1013-1021, September 1988.

[95] M.F.U. Butt, S.X. Ng and L. Hanzo, "Self-Concatenated Code Design and its Application in Power-Efficient Cooperative Communications," IEEE Commun. Surveys \& Tutorials, vol. 14, no. 3, pp. 858-883, 2012.

[96] P. Robertson and T. Worz, "Bandwidth-efficient turbo trellis-coded modulation using punctured component codes," IEEE J. Sel. Areas Commun., vol. 16, pp. 206-218, February 1998.

[97] H. Chen and A. Haimovich, "EXIT charts for turbo trellis-coded modulation," IEEE Commun. Lett., vol. 8, pp. 668-670, November 2004.

[98] T. Yokokawa, Y. Shinohara, T. Miyauchi, Y. Iida and T.J. McEliece, "An optimization method for designing high rate and high performance SCTCM systems with in-line interleavers," in IEEE Global Telecommunications Conference, vol. 1, pp. 162-166, 2004.

[99] S. Benedetto, D. Divsalar, G. Montorsi and F. Pollara, "Selfconcatenated trellis coded modulation with self-iterative decoding," in IEEE Global Telecommunications Conference, vol. 1, pp. 585-591, 1998.

[100] M.F.U. Butt, S.X. Ng and L. Hanzo, "EXIT Chart Aided Design of Near-Capacity Self-Concatenated Trellis Coded Modulation Using Iterative Decoding," in IEEE Vehicular Technology Conference, pp. 734 738, May 2008.

[101] E. Zehavi, "8-PSK trellis codes for a Rayleigh channel," IEEE Trans. Commun., vol. 40, pp. 873-884, May 1992.

[102] X. Li and J.A. Ritcey, "Trellis-coded modulation with bit interleaving and iterative decoding," IEEE J. Sel. Areas Commun., vol. 17, no. 4, pp. 715-724, 1999.

[103] S.X. Ng, J.Y. Chung, P. Cherriman and L. Hanzo, "Burst-by-burst adaptive decision feedback equalized TCM, TTCM, and BICM for H.263-assisted wireless video telephony," IEEE Trans. Circuits Syst. Video Technol., vol. 16, pp. 363-374, March 2006.

[104] C.S. Lee, S.X. Ng, L. Piazzo and L. Hanzo, "TCM, TTCM, BICM and iterative BICM assisted OFDM-based digital video broadcasting to mobile receivers," in IEEE Vehicular Technology Conference, vol. 1, pp. 732-736, 2001.

[105] Y. Huang and J.A. Ritcey, "EXIT chart analysis of BICM-ID with imperfect channel state information," IEEE Commun. Lett., vol. 7, no. 9, pp. 434-436, 2003.

[106] Y. Huang and R.J. Ritcey, "EXIT chart analysis of BICM-ID over AWGN channels with SNR mismatch," IEEE Commun. Lett., vol. 8 , no. 8, pp. 532-534, 2004.

[107] T. Clevorn, S. Godtmann and P. Vary, "BER prediction using EXIT charts for BICM with iterative decoding," IEEE Commun. Lett., vol. 10, no. 1, pp. 49-51, 2006.

[108] R.Y.S. Tee, S.X. Ng and L. Hanzo, "Three-Dimensional EXIT Chart Analys'is of Iteraltive Detecltion Aided Coded Modulaltion Schemes," in IEEE Vehicular Technology Conference, vol. 5, pp. 2494-2498, May 2006.

[109] H. Imai and S. Hirakawa, "A New Multilevel Coding Method Using Error-Correcting Codes," IEEE Trans. Inf. Theory, vol. 23, pp. 784784, November 1977.

[110] R. Tee, R.G. Maunder and L. Hanzo, "EXIT-chart aided near-capacity Irregular Bit-Interleaved Coded Modulation design," IEEE Trans. Wireless Commun., vol. 8, pp. 32-37, January 2009.

[111] Z. Yang, Q. Xie, K. Peng and J. Song, "Labeling Optimization for BICM-ID Systems," IEEE Commun. Lett., vol. 14, pp. 1047-1049, November 2010. 
[112] S. X. Ng, O. Alamri, Y. Li. J. Kliewer and L. Hanzo, "Near-capacity turbo trellis coded modulation design based on EXIT charts and union bounds," IEEE Trans. Commun., vol. 56, pp. 2030-2039, December 2008.

[113] S. Benedetto and G. Montorsi, "Design of parallel concatenated convolutional codes," IEEE Trans. Commun., vol. 44, pp. 591-600, May 1996.

[114] M.S.C. Ho, S.S. Pietrobon and T. Giles, "Improving the constituent codes of turbo encoders," IEEE Global Telecommunications Conference, vol. 6, pp. 3525-3529, 1998.

[115] S. Benedetto, R. Garello and G. Montorsi, "A search for good convolutional codes to be used in the construction of turbo codes," IEEE Trans. Commun., vol. 46, pp. 1101-1105, September 1998.

[116] C. Köse, W.-Y. Weng and R.D. Wesel, "Serially concatenated trellis coded modulation for the compound periodic erasures channel," in IEEE International Conference on Communications, vol. 4, pp. 2953 2957, 2003.

[117] A. Banerjee, F. Vatta, B. Scanavino and D.J. Costello, "Nonsystematic turbo codes," IEEE Trans. Commun., vol. 53, pp. 1841-1849, November 2005 .

[118] C.-Y. Wei, J. Akhtman, S.X. Ng and L. Hanzo, "Iterative NearMaximum-Likelihood Detection in Rank-Deficient Downlink SDMA Systems," IEEE Trans. Veh. Technol., vol. 57, pp. 653-657, January 2008.

[119] V. Kuhn, "Analysis of iterative multi-user detection schemes with EXIT charts," in IEEE Eighth International Symposium on Spread Spectrum Techniques and Applications, pp. 535-539, August-September 2004.

[120] K. Li and X. Wang, "EXIT chart analysis of turbo multiuser detection," IEEE Trans. Wireless Commun., vol. 4, pp. 300-311, January 2005.

[121] R.G. Maunder, J. Wang, S.X. Ng, L.-L. Yang and L. Hanzo, "On the Performance and Complexity of Irregular Variable Length Codes for Near-Capacity Joint Source and Channel Coding," IEEE Trans. Wireless Commun., vol. 7, pp. 1338-1347, April 2008.

[122] R.A. Riaz, R.G. Maunder, M.F.U. Butt, S.X. Ng, S. Chen and L. Hanzo, "EXIT-Chart-Aided Three-Stage Concatenated Ultrawideband Time-Hopping Spread-Spectrum Impulse Radio Design," IEEE Trans. Veh. Technol., vol. 58, pp. 5320-5324, November 2009.

[123] S. Ahmed, R.G. Maunder, L.-L. Yang and L. Hanzo, "Iterative Detection of Unity-Rate Precoded FFH-MFSK and Irregular Variable-Length Coding," IEEE Trans. Veh.Technol., vol. 58, pp. 3765-3770, September 2009.

[124] R.G. Maunder and L. Hanzo, "Near-capacity irregular variable length coding and irregular unity rate coding," IEEE Trans. Wireless Commun., vol. 8, pp. 5500-5507, November 2009.

[125] L. Kong, S.X. Ng, R.G. Maunder and L. Hanzo, "Near-Capacity Cooperative Space-Time Coding Employing Irregular Design and Successive Relaying," IEEE Trans. Commun., vol. 58, pp. 2232-2241, August 2010.

[126] L. Kong, S.X. Ng, R.G. Maunder and L. Hanzo, "MaximumThroughput Irregular Distributed Space-Time Code for Near-Capacity Cooperative Communications," IEEE Trans. Veh. Technol., vol. 59, pp. 1511-1517, March 2010.

[127] H. Chen, R.G. Maunder and L. Hanzo, "Lookup-Table-Based DeferredIteration Aided Low-Complexity Turbo Hybrid ARQ," IEEE Trans. Veh. Technol., vol. 60, pp. 3045-3053, September 2011.

[128] M. El-Hajjar, O. Alamri, R.G. Maunder and L. Hanzo, "Layered Steered Space Time-Spreading-Aided Generalized MC DS-CDMA," IEEE Trans. Veh. Technol., vol. 59, pp. 999-1005, February 2010.

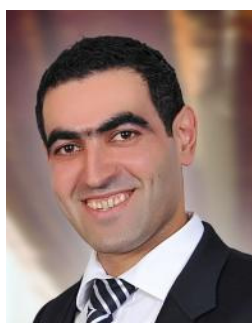

Mohammed El-Hajjar is a lecturer in the Electronics and Computer Science in the University of Southampton. He received his BEng degree in Electrical Engineering from the American University of Beirut, Lebanon in 2004. He then received an MSc in Radio Frequency Communication Systems and $\mathrm{PhD}$ in Wireless Communications both from the University of Southampton, UK in 2005 and 2008, respectively. Following the $\mathrm{PhD}$, he joined Imagination Technologies as a research engineer, where he worked on designing and developing the BICM peripherals in Imagination's multi-standard communications platform, which resulted in several patent applications. In January 2012, he joined the Electronics and Computer Science in the University of Southampton as a lecturer in the Communications, Signal Processing and Control research group. $\mathrm{He}$ is the recipient of several academic awards and has published a Wiley-IEEE book and in excess of 40 journal and international conference papers. His research interests include mm-wave communications, massive MIMO, cooperative communications and Radio over fibre systems.

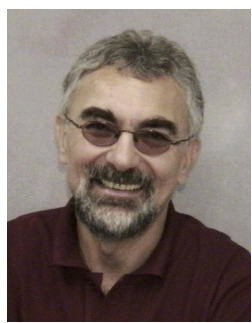

Lajos Hanzo (http://www-mobile.ecs.soton.ac.uk) FREng, FIEEE, FIET, Fellow of EURASIP, DSc received his degree in electronics in 1976 and his doctorate in 1983. In 2009 he was awarded the honorary doctorate "Doctor Honoris Causa" by the Technical University of Budapest. During his 35-year career in telecommunications he has held various research and academic posts in Hungary, Germany and the UK. Since 1986 he has been with the School of Electronics and Computer Science, University of Southampton, UK, where he holds the chair in telecommunications. He has successfully supervised $80 \mathrm{PhD}$ students, co-authored 20 John Wiley/IEEE Press books on mobile radio communications totalling in excess of 10000 pages, published 1300 research entries at IEEE Xplore, acted both as TPC and General Chair of IEEE conferences, presented keynote lectures and has been awarded a number of distinctions. Currently he is directing a 100-strong academic research team, working on a range of research projects in the field of wireless multimedia communications sponsored by industry, the Engineering and Physical Sciences Research Council (EPSRC) UK, the European IST Programme and the Mobile Virtual Centre of Excellence (VCE), UK. He is an enthusiastic supporter of industrial and academic liaison and he offers a range of industrial courses. $\mathrm{He}$ is also a Governor of the IEEE VTS. During 2008 - 2012 he was the Editor-in-Chief of the IEEE Press and since 2009 he has been a Chaired Professor also at Tsinghua University, Beijing. For further information on research in progress and associated publications please refer to http://wwwmobile.ecs.soton.ac.uk 